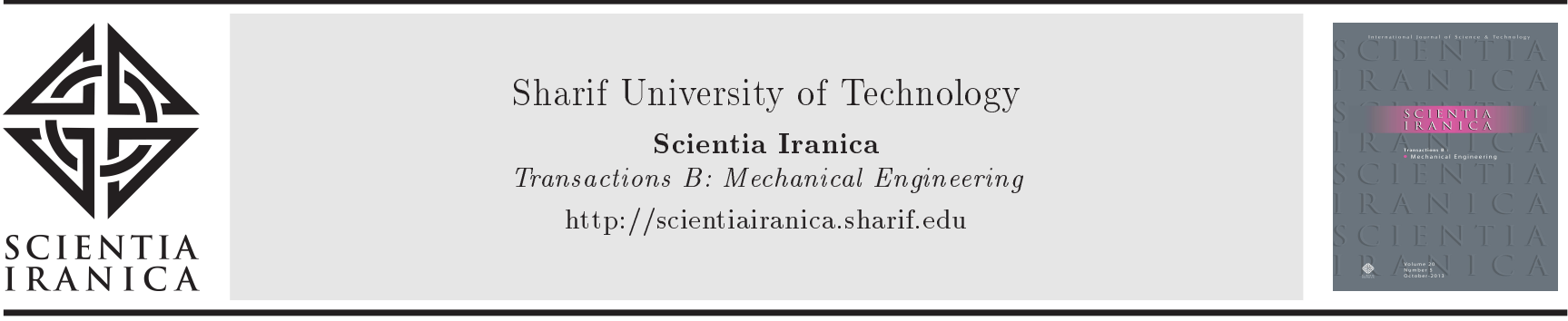

\title{
Numerical investigation into natural convection of nanofluids in an inclined square enclosure with non-uniform heated walls
}

\author{
X. Wang ${ }^{\mathrm{a}, 1, *}$ and W. Dai ${ }^{\mathrm{b}}$ \\ a. Department of Mathematics, Henan Institute of Science and Technology, Xinxiang, Henan 453003, China. \\ b. Mathematics \& Statistics, College of Engineering \& Science, Louisiana Tech University, Ruston, LA 71272, USA. \\ Received 6 February 2016; received in revised form 4 November 2017; accepted 23 April 2018
}

\section{KEYWORDS}

Natural convection;

Inclined enclosure;

Nanofluids;

Sinusoidal

temperature.

\begin{abstract}
Studying natural convection of nanofluids in enclosures with non-uniform heated walls is of importance in many engineering applications such as solar energy collection. In this study, we developed a Fully Higher-Order Compact (FHOC) finite difference method to investigate the natural convection and heat transfer of nanofluids in an inclined square enclosure with sinusoidal temperature distributions. Numerical simulations were performed over a range of amplitude ratios, inclination angles, phase deviations, nanoparticles volume fractions, and Rayleigh numbers. Results showed that heat transfer could increase significantly by increasing the amplitude ratio and inclination angles in nanofluids. Moreover, elevating the nanoparticles volume fraction did not always enhance the heat transfer of nanofluids. When the Rayleigh number $\mathrm{Ra}$ was low $\left(\mathrm{Ra}=10^{3}\right.$ ), the average Nusselt number decreased as the solid volume fraction parameter, $\phi$, increased. On the other hand, elevating $\phi$ had favorable effects on the heat transfer of nanofluids when Ra was high (e.g., $\mathrm{Ra}=10^{4}, 10^{5}$ ). With $\mathrm{Ra}=10^{4}$, the total heat transfer rate decreased with nanoparticles in the order of $\mathrm{Cu}, \mathrm{CuO}, \mathrm{Al}_{2} \mathrm{O}_{3}$, and $\mathrm{TiO}_{2}$. Finally, a correlated expression of the total average Nusselt number, the Rayleigh number, and the solid volume fraction of nanoparticles was empirically obtained.
\end{abstract}

(C) 2019 Sharif University of Technology. All rights reserved.

\section{Introduction}

In nanofluids, which were first introduced by Choi [1], small amounts of metallic or metallic oxide nanoparticles are dispersed into water or other fluids. Recently, there have been tremendous: efforts to identify the mechanisms of convective flow and heat transfer of nanofluids, including the effects of size and shape of

1. Present address: School of Mathematics and Statistics, Minnan Normal University, Zhangzhou, Fujian 363000, PR China.

*. Corresponding author.

E-mail address: wxfmeng@hist.edu.cn (X. Wang)

doi: $10.24200 /$ sci. 2018.20327 nanoparticles, the hydrodynamic interaction between nanoparticles and base fluid, clustering of particles, the influence of temperature or Brownian motion, etc. [2-7]. Heris et al. [8] conducted a comparative experimental study to investigate the effects of metal oxide nanopowders, including $\mathrm{TiO}_{2}, \mathrm{CuO}$, and $\mathrm{Al}_{2} \mathrm{O}_{3}$ suspended in turbine oil, on the natural convection flow inside a titled cube cavity. Rashidi et al. [9] studied the effects of heterogeneous heating on the heat transfer characteristics of a cavity filled with $\mathrm{Al}_{2} \mathrm{O}_{3^{-}}$ water nanofluid. They found that the average Nusselt number increased with increase in the volume fraction of $\mathrm{Al}_{2} \mathrm{O}_{3}$ nanoparticles at $\mathrm{Ra}=10^{3}$. However, for other values of Rayleigh number, there was an optimal value of volume fraction of $\mathrm{Al}_{2} \mathrm{O}_{3}$ nanoparticles in which 
the average Nusselt number was maximized. Wang et al. [10,11] investigated the influence of nanoparticles on the mixed and natural convective heat transfers of nanofluids in square and triangular cavities. They observed that increase in the volume fraction of nanoparticles could enhance the average rate of heat transfer. Mahian et al. [12] considered the case of natural convection of $\mathrm{SiO}_{2}$-water nanofluids in square and triangular cavities and concluded that both theoretical analysis and experimental results predicted the same trend in estimating the average Nusselt number and heat transfer coefficient ratio. Estellé et al. [13] worked on the prediction of heat transfer in a differentially heated square cavity filled with Newtonian or nonNewtonian carbon nanotube nanofluids. Their results mainly proved that the Nusselt number of nanofluids, unlike thermal conductivity, was lowered by increase in nanoparticle content due to non-Newtonian behaviour of nanofluids and temperature increase. Alizadeh and Dehghan [14] conducted a numerical investigation to explore the flow and thermal fields of conjugate natural convection of nanofluids within a square cavity. They concluded that nanofluid type was a key factor in heat transfer enhancement, and $\mathrm{CuO}$ nanoparticles led to a better heat transfer rate than $\mathrm{Al}_{2} \mathrm{O}_{3}$ nanoparticles did. Cianfrini et al. [15] numerically studied the laminar natural convection of $\mathrm{Al}_{2} \mathrm{O}_{3}$-water nanofluids inside square cavities. They found that when the nanoparticle volume fraction increased to an optimal particle loading, the amount of heat transfer of nanofluids reached a peak across the enclosure.

However, whether the rate of heat transfer can be enhanced by means of nanofluids is still an interesting question [16]. Contradictory studies indicate that the presence of nanoparticles in the base fluid may result in a considerable decrease in the heat transfer [17]. Mahmoudi et al. [18] numerically examined how the position of horizontal heat source on the left vertical wall would affect the heat transfer in the cavity filled with $\mathrm{Cu}$-water nanofluids. They found that locating the heat source close to the top horizontal wall would lead to more effective outcomes in the heat transfer of nanofluids. Haddad et al. [19] studied the natural convection heat transfer of $\mathrm{CuO}$-water nanofluids. They found that the enhancement in heat transfer was more pronounced at low volume fractions of nanoparticles and the heat transfer decreased with increase in the volume fraction of nanoparticles.

Up to date, most of the research studies on natural convection and heat transfer of nanofluids in enclosures reported in the literature have been concerned with fully heated cavities [20-22]. But, in realistic situations, the active walls may be subject to nonuniform temperature distribution due to shadows and other natural interferences, such as solar energy collection [23]. It is important to apply solar energy to a wide range of applications and provide solutions through the modification of the energy proportion, improving energy stability, increasing energy sustainability, and enhancing system efficiency [24]. Mahian et al. [25] investigated the effects of nanoparticle suspensions on the performance of a solar still equipped with a heat exchanger, both experimentally and theoretically. It was found that in high temperatures, using $\mathrm{SiO}_{2}$-water nanofluids, which had lower effective thermal conductivity than $\mathrm{Cu}$-water nanofluids, provided higher performance indices. Usually, the solar collector has flat plate cover and sinusoidal wavy absorber [26]. Thus, investigating the convective heat transfer in enclosures with sinusoidal heated walls is very helpful in such situations. Arani et al. [27] investigated the mixed convection and heat transfer of $\mathrm{Cu}$-water nanofluid inside a lid-driven square cavity with sinusoidal temperature distribution on sidewalls. They showed that when the Richardson number decreased or the volume faction of nanoparticles increased, the rate of heat transfer could increase. Nasrin and Alim [28] compared performances of different nanofluids for the natural convective flow inside a solar collector with a sinusoidal wave absorber. They found better performance in heat transfer inside the collector could be achieved by the highest volume fraction of Ag nanoparticles. Mejri and Mahmoud [29] presented a numerical study to examine the natural convection in an open cavity with a sinusoidal thermal boundary condition. Their study revealed that the heat transfer rate increased with increase in the Rayleigh number.

However, review of the literature indicates that few studies have been done on natural convection and heat transfer in an inclined square enclosure subjected to sinusoidal temperature distribution on boundary for nanofluids. Hence, the objective of this paper is to numerically investigate such case, in order to see the effects of the sinusoidal boundary conditions at horizontal sidewalls on natural convection and heat transfer in different types of nanofluids in an inclined square enclosure. In addition, we will establish a general expression for the correlation of the total average Nusselt number, the Rayleigh number, and the solid volume fraction of nanoparticles.

\section{Mathematical formulation}

We consider the natural convection and heat transfer of water-based nanofluids in an inclined square enclosure with non-uniform heating on both top and bottom sidewalls, as shown in Figure 1. The width and height of the inclined square enclosure are assumed to be $L$. The left and right walls of the enclosure are adiabatic. The top and bottom horizontal sidewalls are kept with sinusoidal temperature distributions [22], where $T_{t}$ and $T_{b}$ are temperatures of the top and 


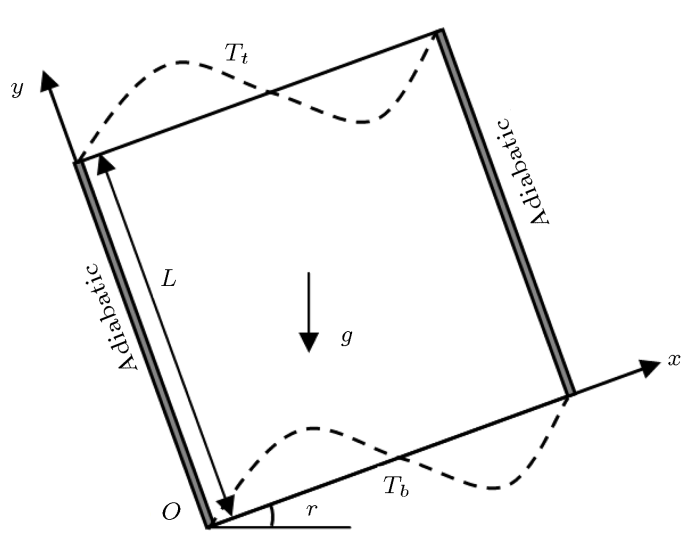

Figure 1. Schematic diagram of the enclosure.

bottom walls, respectively. It should be pointed out that we impose a surface temperature distribution in order to simplify the problem. Such type of surface distribution can be used to model the effects of a periodic array of heaters behind or within the top and bottom walls [30]. Here, we consider four different types of nanoparticles, namely $\mathrm{Cu}, \mathrm{CuO}, \mathrm{Al}_{2} \mathrm{O}_{3}$, and $\mathrm{TiO}_{2}$; their thermo-physical properties are listed in Table 1 [31]. For simplicity, we assume the convective flow to be two-dimensional, Newtonian, steady, and incompressible [31]. It is further assumed that the base fluid (pure water) and nanoparticles are in thermal equilibrium; no slip occurs between these two media; and they flow with the same local velocity. In addition, we assume that the thermo-physical properties of the nanofluids are constant except for the density variation approximated by the Boussinesq model [32]. Thus, based on the above assumptions, the mathematical model governing the natural convection and heat transfer of nanofluids in an inclined square enclosure with non-uniform heating can be expressed as follows $[31,32]$ :

$$
\begin{aligned}
& \frac{\partial u}{\partial x}+\frac{\partial v}{\partial y}=0 \\
& u \frac{\partial u}{\partial x}+v \frac{\partial u}{\partial y}=-\frac{1}{\rho_{n f}} \frac{\partial p}{\partial x} \\
& \quad+\frac{\mu_{n f}}{\rho_{n f}} \nabla^{2} u+\frac{(\rho \beta)_{n f}}{\rho_{n f}} g \sin (r)\left(T-T_{c}\right),
\end{aligned}
$$

$$
\begin{aligned}
& u \frac{\partial v}{\partial x}+v \frac{\partial v}{\partial y}=-\frac{1}{\rho_{n f}} \frac{\partial p}{\partial y} \\
&+\frac{\mu_{n f}}{\rho_{n f}} \nabla^{2} v+\frac{(\rho \beta)_{n f}}{\rho_{n f}} g \cos (r)\left(T-T_{c}\right), \\
& u \frac{\partial T}{\partial x}+v \frac{\partial T}{\partial y}=\alpha_{n f} \nabla^{2} T .
\end{aligned}
$$

Here, $u, v$ are components of the velocity in $x$ - and $y$ directions, respectively, $p$ is pressure, $T$ is temperature, and $r$ is the inclination angle. The water-based nanofluid is idealized as a single-phase fluid. Hence, the equations of physical parameters of nanofluids are given as follows [33-36]. The effective density of nanofluid is $\rho_{m}=(1-\phi) \rho_{f}+\phi \rho_{s}$, the thermal diffusivity of nanofluid is $\alpha_{m}=\kappa_{m} /\left(\rho c_{p}\right)_{m}$, where $\left(\rho c_{p}\right)_{m}$ is the heat capacitance of nanofluid given by $\left(\rho c_{p}\right)_{m}=$ $(1-\phi)\left(\rho c_{p}\right)_{f}+\phi\left(\rho c_{p}\right)_{s}$, and the thermal expansion coefficient of nanofluid is $(\rho \beta)_{m}=(1-\phi)(\rho \beta)_{f}+$ $\phi(\rho \beta)_{s}$. The viscosity of nanofluid is assumed to follow the Brinkman relation as [32] $\mu_{m}=\mu_{f}(1-\phi)^{-2.5}$. The effective thermal conductivity of nanofluid is approximated by the Maxwell-Garnetts model as [37]:

$$
\begin{array}{r}
\kappa_{m}=\kappa_{f}\left(\kappa_{s}+2 \kappa_{f}-2 \phi\left(\kappa_{f}-\kappa_{s}\right)\right) \\
\left(\kappa_{s}+2 \kappa_{f}+\phi\left(\kappa_{f}-\kappa_{s}\right)\right)^{-1}
\end{array}
$$

The corresponding boundary conditions are described as follows: $u=v=0$ on all the four walls, $\partial T / \partial x=$ 0 on the vertical walls at $x=0$ and $x=L, T_{b}=$ $T_{c}+A_{b} \sin (2 \pi x / L+\iota)$ on the bottom wall and $T_{t}=$ $T_{c}+A_{t} \sin (2 \pi x / L)$ on the top wall, where $A_{t}$ and $A_{b}$ are amplitudes of the sinusoidal temperature on top and bottom walls of the enclosure, respectively, and the phase deviation of the sinusoidal temperature of the bottom wall is $\iota$ [38].

In order to rewrite Eqs. (1)-(4) in a dimensionless form, we introduce the following variables:

$$
\begin{aligned}
& X=\frac{x}{L}, \quad Y=\frac{y}{L}, \quad U=\frac{u L}{\alpha_{f}}, \quad V=\frac{v L}{\alpha_{f}}, \\
& \theta=\frac{T-T_{c}}{\Delta T}, \quad P=\frac{p L^{2}}{\rho_{n f} \alpha_{f}^{2}} .
\end{aligned}
$$

Table 1. Thermo-physical properties of water and nanoparticles.

\begin{tabular}{lccccc}
\hline Property & Water & $\mathbf{C u}$ & $\mathbf{C u O}$ & $\mathbf{A l}_{\mathbf{2}} \mathbf{O}_{\mathbf{3}}$ & $\mathbf{T i O}_{\mathbf{2}}$ \\
\hline$\rho\left(\mathrm{kg} / \mathrm{m}^{3}\right)$ & 997.1 & 8933 & 6500 & 3970 & 4250 \\
$c_{p}\left(\mathrm{Jkg}^{-1} \mathrm{~K}^{-1}\right)$ & 4179 & 385 & 535.6 & 765 & 686.2 \\
$k\left(\mathrm{Wm}^{-1} \mathrm{~K}^{-1}\right)$ & 0.613 & 400 & 20 & 40 & 8.9538 \\
$\beta\left(\mathrm{K}^{-1}\right)$ & 0.00021 & 0.000051 & 0.000051 & 0.000024 & 0.000024 \\
\hline
\end{tabular}


Thus, the dimensionless form of stream function and vorticity formulation can be written as follows:

$$
\begin{aligned}
& \frac{\partial^{2} \Phi}{\partial X^{2}}+ \frac{\partial^{2} \Phi}{\partial Y^{2}}=-\Omega, \\
& U \frac{\partial \Omega}{\partial X}+ V \frac{\partial \Omega}{\partial Y}=\frac{\mu_{n f}}{\rho_{n f} \alpha_{f}} \nabla^{2} \Omega \\
&+\frac{(\rho \beta)_{n f}}{\rho_{n f} \beta_{f}} \operatorname{RaPr}\left(\cos (r) \frac{\partial \theta}{\partial X}-\sin (r) \frac{\partial \theta}{\partial Y}\right), \\
& U \frac{\partial \theta}{\partial X}+V \frac{\partial \theta}{\partial Y}=\frac{\alpha_{n f}}{\alpha_{f}} \nabla^{2} \theta,
\end{aligned}
$$

where $\Phi, \Omega$, and $\theta$ are non-dimensional stream function, vorticity, and temperature, respectively, Ra is the Rayleigh number which is defined as $\mathrm{Ra}=$ $g \beta_{f} L^{3} \Delta T /\left(\gamma_{f} \alpha_{f}\right)$, and $\operatorname{Pr}$ is the Prandtl number for the base fluid, which is defined as $\operatorname{Pr}=\nu_{f} / \alpha_{f}$ [31].

The corresponding non-dimensional boundary conditions are as follows: $\Phi=0$ on all the four walls, $\Omega=-\partial^{2} \Phi / \partial X^{2}$ and $\partial \theta / \partial X=0$ on the left and right walls $X=0$ and $X=1, \Omega=-\partial^{2} \Phi / \partial Y^{2}$ and $\theta=\varepsilon \sin (2 \pi X+\iota)$ on the bottom wall $Y=0$, $\Omega=-\partial^{2} \Phi / \partial Y^{2}$ and $\theta=\sin (2 \pi X)$ on the top wall $Y=1$, where $\varepsilon=A_{b} / A_{t}$ is the amplitude ratio of the sinusoidal temperature on the bottom wall to that on the top wall of the enclosure. The local Nusselt numbers along the isothermal walls of the enclosure are expressed as follows [32]:

$\mathrm{Nu}_{b}(X)=-\left.\frac{\kappa_{m}}{\kappa_{f}} \frac{\partial \theta}{\partial Y}\right|_{Y=0}, \quad \mathrm{Nu}_{t}(X)=-\left.\frac{\kappa_{m}}{\kappa_{f}} \frac{\partial \theta}{\partial Y}\right|_{Y=1}$

The averaged Nusselt numbers along the horizontal sidewalls are expressed as follows [22]:

$$
\begin{aligned}
& \mathrm{Nu}(b)=\int_{0}^{1} \mathrm{Nu}_{b}(X) d X, \\
& \mathrm{Nu}(t)=\int_{0}^{1} \mathrm{Nu}_{t}(X) d X .
\end{aligned}
$$

Furthermore, $\mathrm{Nu}_{\text {avg }}=\mathrm{Nu}(b)+\mathrm{Nu}(t)$ is the total average Nusselt number across the entire enclosure. The above systems in Eqs. (7)-(9) and the corresponding dimensionless boundary conditions are complicated, and the analytic solution may be difficult to find. Therefore, we will carry out a numerical simulation in the next section.

\section{Numerical simulation}

To develop a higher-order accurate numerical method for solving the above heat transfer and convective flow of nanofluids in an inclined square enclosure with nonuniform heating, we first design a mesh as $X_{i}=i h$, $Y_{j}=j h, h=1 / M, i, j=0,1, \ldots, M$, where $M$ is a positive integer, $h$ is the mesh size, and $\psi_{i, j}$ denotes the approximation of the function $\psi\left(X_{i}, Y_{j}\right)$, $\psi=\Phi, \Omega, \theta$. We also note that each of Eqs. (7)-(9) can be viewed as the following steady-state convection diffusion equation [39]:

$-\frac{\partial^{2} \psi}{\partial X^{2}}-\frac{\partial^{2} \psi}{\partial Y^{2}}+a(X, Y) \frac{\partial \psi}{\partial X}+b(X, Y) \frac{\partial \psi}{\partial Y}=s(X, Y)$,

where $\psi$ is a transport variable representing $\Phi, \Omega$, and $\theta$, respectively; and $a, b$, and $s$ denote the corresponding coefficient functions with respect to $x$ and $y$. We start by examining the $1 \mathrm{D}$ steady convection diffusion equation:

$$
-\frac{d^{2} \psi}{d X^{2}}+a(X) \frac{d \psi}{d X}=s(X) .
$$

For the numerical solution to Eq. (13), the following second-order $O\left(h^{2}\right)$ accurate finite difference scheme is employed:

$$
-\delta_{X}^{2} \psi_{i}+a_{i} \delta_{X} \psi_{i}=s_{i}, \quad i=0,1,2, \ldots, M,
$$

where $\delta_{X}$ and $\delta_{X}^{2}$ are the first- and second-order central difference operators in the $X$-direction, respectively, such that:

$$
\begin{aligned}
\delta_{X} \psi_{i} & =\left(\psi_{i+1}-\psi_{i-1}\right) /(2 h), \\
\delta_{X}^{2} \psi_{i} & =\left(a_{i+1}-2 a_{i}+a_{i, j}\right) / h^{2} .
\end{aligned}
$$

To establish a higher-order accurate spatial compact finite difference approximation of Eq. (13), the finite difference approximations of the first and second derivatives appearing in Eq. (13) are given as follows:

$$
\begin{aligned}
& \frac{d \psi}{d X}=\delta_{X} \psi-\frac{h^{2}}{6} \frac{d^{3} \psi}{d X^{3}}+O\left(h^{4}\right), \\
& \frac{d^{2} \psi}{d X^{2}}=\delta_{X}^{2} \psi-\frac{h^{2}}{12} \frac{d^{4} \psi}{d X^{4}}+O\left(h^{4}\right) .
\end{aligned}
$$

Substituting Eq. (15) into Eq. (14), we obtain:

$$
-\delta_{X}^{2} \psi_{i}+a_{i} \delta_{X} \psi_{i}-\frac{a_{i}}{6} h^{2} \frac{d^{3} \psi_{i}}{d X^{3}}+\frac{h^{2}}{12} \frac{d^{4} \psi_{i}}{d X^{4}}+O\left(h^{4}\right)=s_{i} .
$$

By taking the first and second derivatives $(\partial / \partial X$, $\left.\partial^{2} / \partial X^{2}\right)$ to Eq. (13), respectively, we obtain:

$$
\begin{aligned}
\frac{d^{3} \psi}{d X^{3}}= & a \frac{\partial^{2} \psi}{d X^{2}}+\frac{d a}{d X} \frac{d \psi}{d X}-\frac{d s}{d X}, \\
\frac{d^{4} \psi}{d X^{4}}= & \left(a^{2}+2 \frac{d a}{d X}\right) \frac{d^{2} \psi}{d X^{2}}+\left(\frac{d^{2} a}{d X^{2}}\right. \\
& \left.+a \frac{d a}{d X}\right) \frac{d \psi}{d X}-a \frac{d s}{d X}-\frac{d^{2} s}{d X^{2}} .
\end{aligned}
$$


First, discretizing the right-hand-sides in Eqs. (17) and (18) by using the first- and second-order central difference operators, respectively, and then, substituting them into Eq. (16), we obtain a spatially fourth-order accurate compact approximation of Eq. (13) as:

$$
-A_{i} \delta_{X}^{2} \psi_{i}+C_{i} \delta_{X} \psi_{i}=S_{i}
$$

where the corresponding coefficients are:

$$
\begin{aligned}
& A_{i}=1+\varpi\left(a^{2}-2 \delta_{X} a\right)_{i}, \\
& C_{i}=a_{i}+\varpi\left(\delta_{X}^{2} a-a \delta_{X} a\right)_{i}, \\
& S_{i}=s_{i}+\varpi\left(\delta_{X}^{2} s-a \delta_{X} s\right)_{i},
\end{aligned}
$$

and $\varpi=h^{2} / 12$.

We now extend the above fourth-order compact method for the $1 \mathrm{D}$ convection diffusion problems to the $2 \mathrm{D}$ case. The $2 \mathrm{D}$ convection diffusion equation in Eq. (12) is first split into the following two systems:

$$
\begin{aligned}
& \left\{\begin{array}{l}
-\frac{\partial^{2} \psi}{\partial X^{2}}+a(X, Y) \frac{\partial \psi}{\partial X}=s_{1}(X, Y), \\
s_{1}(X, Y)=\frac{\partial^{2} \psi}{\partial Y^{2}}-b(X, Y) \frac{\partial \psi}{\partial Y}+s(X, Y),
\end{array}\right. \\
& \left\{\begin{array}{l}
-\frac{\partial^{2} \psi}{\partial Y^{2}}+b(X, Y) \frac{\partial \psi}{\partial Y}=s_{2}(X, Y), \\
s_{2}(X, Y)=\frac{\partial^{2} \psi}{\partial X^{2}}-a(X, Y) \frac{\partial \psi}{\partial X}+s(X, Y) .
\end{array}\right.
\end{aligned}
$$

Applying Eqs. (19) and (20) to the 1D-like Eqs. (21) and (22), we obtain the spatially fourth-order compact approximations of Eq. (12) as:

$-A_{i, j}^{\psi} \delta_{X}^{2} \psi_{i, j}-B_{i, j}^{\psi} \delta_{Y}^{2} \psi_{i, j}+C_{i, j}^{\psi} \delta_{X} \psi_{i, j}+D_{i, j}^{\psi} \delta_{Y} \psi_{i, j}=S_{i, j}^{\psi}$,

where the coefficients are given as follows:

$$
\begin{aligned}
A_{i, j}^{\psi}= & 1+\varpi\left(a^{2}-2 \delta_{X} a\right)_{i, j}, \\
B_{i, j}^{\psi}= & 1+\varpi\left(b^{2}-2 \delta_{Y} b\right)_{i, j}, \\
C_{i, j}^{\psi}= & a_{i, j}+\varpi\left(\delta_{X}^{2} a+\delta_{Y}^{2} a-a \delta_{X} a-b \delta_{Y} a\right)_{i, j}, \\
D_{i, j}^{\psi}= & b_{i, j}+\varpi\left(\delta_{X}^{2} b+\delta_{Y}^{2} b-a \delta_{X} b-b \delta_{Y} b\right)_{i, j}, \\
S_{i, j}^{\psi}= & s_{i, j}+\varpi\left(\delta_{X}^{2} s+\delta_{Y}^{2} s-a \delta_{X} s\right. \\
& \left.-b \delta_{Y} s+2 \Upsilon\right)_{i, j}, \\
\Upsilon= & \delta_{X}^{2} \delta_{Y}^{2} \psi-a \delta_{X} \delta_{Y}^{2} \psi-b \delta_{X}^{2} \delta_{Y} \psi \\
& -\delta_{X} \delta_{Y} \psi\left(\delta_{Y} a+\delta_{X} b-a b\right) .
\end{aligned}
$$

The standard second-order central difference operators within the nine-point stencil are given in Appendix A.
Note that one has to solve three systems based on Eqs. (23)-(29) for stream function, vorticity, and temperature. To simplify the computation, we introduce the pesudo-time algorithm [40,41] for solving Eqs. (23)(29):

$$
\begin{aligned}
\partial \psi_{i, j}^{n} / \partial t= & -A_{i, j}^{\psi} \delta_{X}^{2} \psi_{i, j}^{n}-B_{i, j}^{\psi} \delta_{Y}^{2} \psi_{i, j}^{n}+C_{i, j}^{\psi} \delta_{X} \psi_{i, j}^{n} \\
& +D_{i, j}^{\psi} \delta_{Y} \psi_{i, j}^{n}-S_{i, j}^{\psi} .
\end{aligned}
$$

As a result, at steady state, $\psi^{n+1}$ converges to $\psi^{n}$, so that $\partial \psi^{n} / \partial t \approx 0$, and the final solution to Eq. (30) converges to the solution to Eq. (23). We use the Alternating Directing Implicit (ADI) method to solve Eq. (30) until the solution converges to the steady state.

Finally, the computation procedure of the FHOC scheme for obtaining the stream function, vorticity, and temperature in the nanofield problem can be described as:

Step 1. Solve the stream function based on the FHOC scheme for Eq. (7). To this end, determine stream function boundaries on the walls based on $\mathbf{U}=0$ and obtain $\Phi^{n+1}=0$. Then, use the ADI method to solve the following equation in region $[1, M-1] \times[1, M-1]$ to obtain $\Phi^{n+1}$ :

$$
\begin{aligned}
\partial \Phi_{i, j}^{n} / \partial t= & -A_{i, j}^{\Phi} \delta_{X}^{2} \Phi_{i, j}^{n}-B_{i, j}^{\Phi} \delta_{Y}^{2} \Phi_{i, j}^{n} \\
& +C_{i, j}^{\Phi} \delta_{X} \Phi_{i, j}^{n}+D_{i, j}^{\Phi} \delta_{Y} \Phi_{i, j}^{n}-S_{i, j}^{\Phi},
\end{aligned}
$$

where,

$$
\begin{aligned}
& A_{i, j}^{\Phi}=1, \quad B_{i, j}^{\Phi}=1, \quad C_{i, j}^{\Phi}=0, \quad D_{i, j}^{\Phi}=0, \\
& S_{i, j}^{\Phi}=\Omega_{i, j}+\varpi\left(\delta_{X}^{2} \Omega+\delta_{Y}^{2} \Omega+\delta_{X}^{2} \delta_{Y}^{2} \Phi\right)_{i, j} .
\end{aligned}
$$

Step 2. Solve the vorticity based on the FHOC scheme for Eq. (8). To this end, first determine vorticity boundaries on the walls. The boundaries on the bottom wall are given as:

$$
\begin{aligned}
\Omega_{i, 0}^{n+1}= & -\frac{h}{12} \chi_{1}\left[2 \cos (r)\left(\theta_{i, 1}^{n}-\theta_{i, 0}^{n}\right)\right. \\
& \left.-\sin (r)\left(\theta_{i+1,0}^{n}-\theta_{i-1,0}^{n}\right)\right]-\frac{4}{h^{2}} \Phi_{i, 1}^{n+1}-\chi_{2}^{n},
\end{aligned}
$$

where:

$$
\begin{aligned}
& \chi_{1}=\operatorname{Ra} \operatorname{Pr}\left[(\rho \beta)_{n f} \alpha_{f}\right] /\left(\mu_{f} \beta_{f}\right), \\
& \chi_{2}^{n}=2 \Omega_{i, 1}^{n} / 3+\left(\Omega_{i+1,0}^{n}+\Omega_{i-1,0}^{n}\right) / 6,
\end{aligned}
$$

and similarly for the other three walls. Then, solve the following equation:

$$
\begin{aligned}
\partial \Omega_{i, j}^{n} / \partial t= & -A_{i, j}^{\Omega} \delta_{X}^{2} \Omega_{i, j}^{n}-B_{i, j}^{\Omega} \delta_{Y}^{2} \Omega_{i, j}^{n} \\
& +C_{i, j}^{\Omega} \delta_{X} \Omega_{i, j}^{n}+D_{i, j}^{\Omega} \delta_{Y} \Omega_{i, j}^{n}-S_{i, j}^{\Omega}
\end{aligned}
$$

in region $[1, M-1] \times[1, M-1]$ to obtain $\Omega^{n+1}$. 


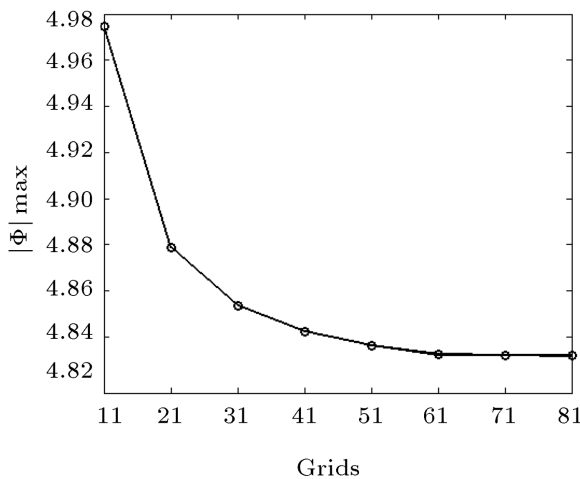

(a)

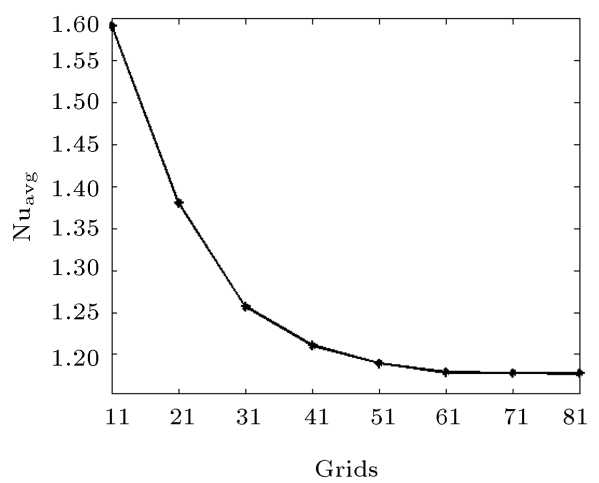

(b)

Figure 2. Grid independence study based on (a) the maximum value of the stream function and (b) the total average Nusselt number across the entire inclined square enclosure as a function of the grid size for the natural convection flow of Cu-water nanofluid.

Step 3. Solve the temperature based on the FHOC scheme for Eq. (9). First, determine the temperature boundaries on the left-hand-side wall based on $\partial \theta / \partial X=0$, and then develop a fourth-order discrete temperature boundary condition on $X=0$ as:

$\theta_{0, j}^{n+1}=\left[\theta_{1, j+1}^{n}+\theta_{1, j-1}^{n}+2 \theta_{0, j+1}^{n}+4 \theta_{1, j}^{n}+2 \theta_{0, j-1}^{n}\right] / 10$.

Finally, solve the temperature equation:

$$
\begin{aligned}
\partial \theta_{i, j}^{n} / \partial t= & -A_{i, j}^{\theta} \delta_{X}^{2} \theta_{i, j}^{n}-B_{i, j}^{\theta} \delta_{Y}^{2} \theta_{i, j}^{n} \\
& +C_{i, j}^{\theta} \delta_{X} \theta_{i, j}^{n}+D_{i, j}^{\theta} \delta_{Y} \theta_{i, j}^{n}-S_{i, j}^{\theta},
\end{aligned}
$$

in region $[1, M-1] \times[1, M-1]$ to obtain $\Omega^{n+1}$.

Step 4. Repeat Step 1 to Step 3 from $n=0,1,2, \ldots$, until the following convergence criterion for stream function, $\Phi$, vorticity, $\Omega$, and temperature, $\theta$, is satisfied:

$$
\begin{aligned}
& \max \left(\left|\Phi^{n+1}-\Phi^{n}\right|\right) \leq \xi, \\
& \max \left(\left|\Omega^{n+1}-\Omega^{n}\right|\right) \leq \xi, \\
& \max \left(\left|\theta^{n+1}-\theta^{n}\right|\right) \leq \xi,
\end{aligned}
$$

where $\xi=10^{-6}$ in our computation.

\section{Numerical results}

We first tested the grid independence of the present
FHOC scheme. To this end, eight different meshes of $11 \times 11,21 \times 21,31 \times 31,41 \times 41,51 \times 51,61 \times 61,71 \times 71$, and $81 \times 81$ were used for the natural convection and heat transfer of $\mathrm{Cu}$-water nanofluid. The maximum value of the stream function of the primary eddy $|\Phi|_{\max }$ and the total average Nusselt number $\mathrm{Nu}_{\text {avg }}$ across the entire inclined square enclosure were used to measure the accuracy of the solution [42]. Figure 2 demonstrates the influence of the number of grid points on natural convection and heat transfer of $\mathrm{Cu}$-water nanofluid, where $\operatorname{Pr}=6.8, \xi=10^{-6}, \mathrm{Ra}=10^{4}, \varepsilon=0.5, \iota=90^{\circ}$, $\phi=5 \%$, and $r=30^{\circ}$. It can be seen from Figure 2 that when the mesh is finer, the solution is more accurate; and when the mesh is finer than $61 \times 61$, the solution does not change significantly. This indicates that our scheme is grid-independent. We then tested our FHOC scheme for the classical natural convection heat transfer of pure water in a differentially heated square enclosure, where $\mathrm{Ra}=10^{3}$. The obtained numerical results were compared with those given by various references listed in Table 2. As seen from Table 2, the obtained numerical results show a good agreement with those given by the existing references [43-49]. Furthermore, our numerical simulation procedure was tested in a square enclosure where the $\mathrm{Al}_{2} \mathrm{O}_{3}$-water nanofluid was filled and the bottom wall was kept with a non-uniform temperature distribution as that in [50], where $\mathrm{Ra}=$ $10^{5}, \operatorname{Pr}=6.2$, and $\phi=10 \%$. The obtained averaged

Table 2. Comparison of the average Nusselt numbers of pure fluid in different references when $\mathrm{Ra}=10^{3}$.

\begin{tabular}{lccc}
\hline \multicolumn{1}{c}{ References } & Nu & References & Nu \\
\hline Bejan [43] & 15.800 & Baytas and Pop [47] & 14.060 \\
Gross et al. [44] & 13.448 & Saeid and Pop [48] & 13.726 \\
Manole and Lage [45] & 13.637 & Varol et al. [49] & 13.564 \\
Goyeau et al. [46] & 13.470 & This study & 13.612 \\
\hline
\end{tabular}


Nusselt number through the hot source is 6.955 , which is in an excellent agreement with 6.956 obtained in [51].

We then investigated the natural convection and heat transfer in an inclined square enclosure utilizing nanofluids with four types of nanoparticles $(\mathrm{Cu}, \mathrm{CuO}$, $\mathrm{Al}_{2} \mathrm{O}_{3}, \mathrm{TiO}_{2}$ )-water with non-uniform heating. Numerical results were studied based on the following parameters: the Rayleigh number $\left(\mathrm{Ra}=10^{3}, 10^{4}, 10^{5}\right)$, the enclosure inclination angle ) $\left.r=0^{\circ}, 30^{\circ}, 45^{\circ}\right)$, the solid volume fractions $(\phi=0 \%, 1 \%, 2 \%, 3 \%, 4 \%, 5 \%)$, the amplitude ratio of the sinusoidal temperature $(\varepsilon=0,0.5,1.0)$, and the phase deviation of the sinusoidal temperature of the bottom wall $(\iota=$ $0, \pi / 4, \pi / 2,3 \pi / 4, \pi)$. Throughout the study, $\operatorname{Pr}=6.8$ and $\xi=10^{-6}$ were fixed.

Figure 3 illustrates the local Nusselt number along
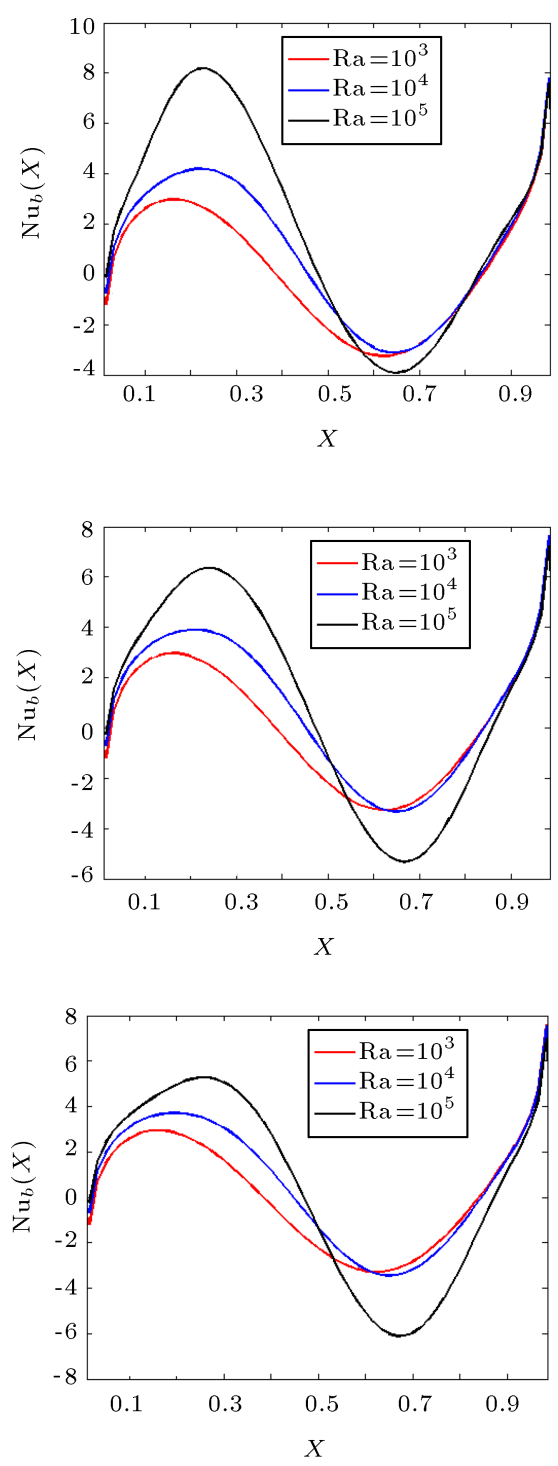

(b)

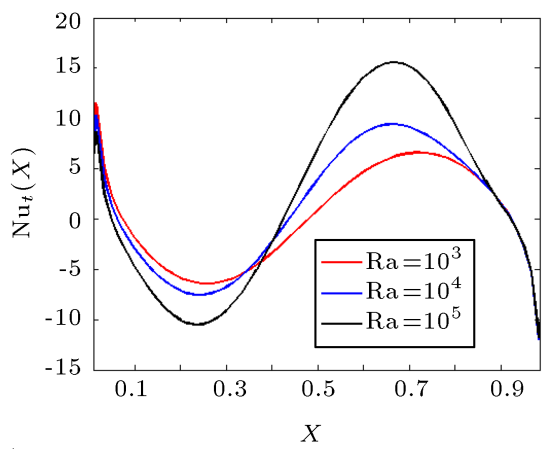

(a)

the bottom wall (on the left column) and the top wall (on the right column) for different Rayleigh numbers and inclination angles of $\mathrm{CuO}$-water nanofluid, where $\phi=5 \%, \varepsilon=0.5$, and $\iota=\pi / 4$. One may observe from these figures that the local Nusselt number $\mathrm{Nu}_{b}(X)$ along the bottom wall increases as Ra increases on the left half of the enclosure $(0 \leq X \leq 0.5)$; on the other hand, it decreases as $\mathrm{Ra}$ increases on the right half of the enclosure $(0.5 \leq X \leq 1)$. Furthermore, the local Nusselt number $\mathrm{Nu}_{t}(X)$ along the top wall decreases as Ra, increases on the left half $(0 \leq X \leq 0.4)$, and increases as $\mathrm{Ra}$ increases on the right half $(0.4 \leq X \leq$ 1). In addition, when the Rayleigh number is low ( $\mathrm{Ra}$ $=10^{3}$ ), changing the inclination angle, $r$, along both the bottom and top walls has no effects on the heat transfer rate. When the Rayleigh number, $\mathrm{Ra}$, is high
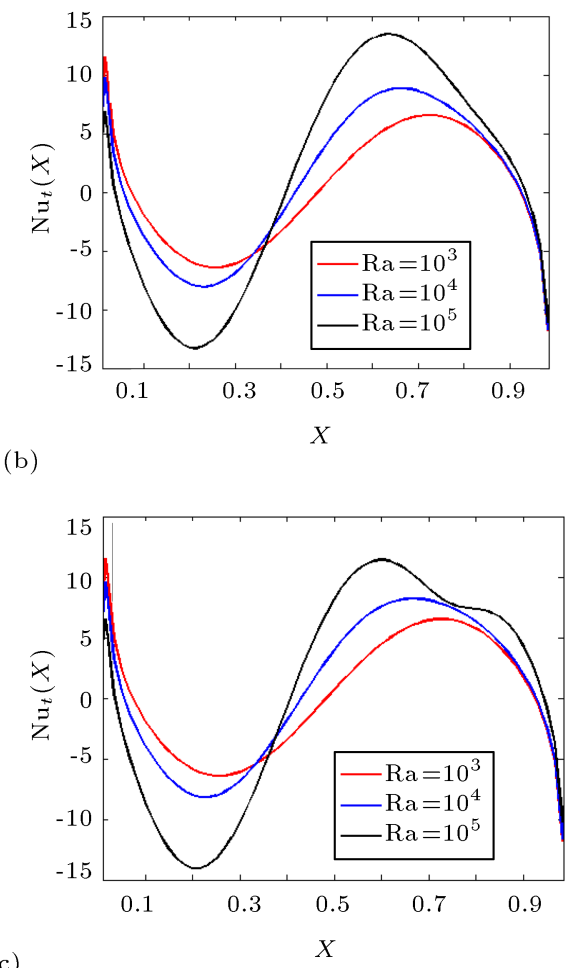

Figure 3. Local Nusselt numbers along the bottom wall (on the left column) and the top wall (on the right column) for different Rayleigh numbers at (a) $r=0^{\circ}$, (b) $r=30^{\circ}$, and (c) $r=45^{\circ}$ for the CuO-water nanofluid. 
$\left(\mathrm{Ra}=10^{4}, 10^{5}\right)$, the heat transfer rate decreases as the inclination angle increases from $0^{\circ}$ to $45^{\circ}$. These observations are similar to those of Varol et al. [49] for a pure fluid case.

Figure 4 shows the local Nusselt number $\mathrm{Nu}_{b}$ along the bottom wall of the inclined square enclosure for different amplitude ratios, $\varepsilon$, for $\mathrm{Al}_{2} \mathrm{O}_{3}$-water nanofluids with $\phi=5 \%, r=30^{\circ}$, and $\iota=\pi / 4$. From Figure 4, one may observe that heat transfer rate, $\mathrm{Nu}_{b}(X)$, along the bottom wall increases as the amplitude ratio, $\varepsilon$, increases on the left half $(0 \leq$ $X \leq 0.4$ ) and in a narrow zone close to the right wall $(0.8 \leq X \leq 1)$; on the other hand, it decreases as the amplitude ratio, $\varepsilon$, increases on the right half $(0.5 \leq X \leq 0.8)$. This is because the temperature $\theta$ on the bottom boundary is $\varepsilon \sin (2 \pi X+\iota)$; and $\varepsilon$ is dependent only on the bottom wall and the energy transport on the top wall is not affected by it. As a result, changing $\varepsilon$ will not affect the heat transfer rate, $\mathrm{Nu}_{t}(X)$, along the top wall. These observations are similar to those of Sivasankaran et al. [22].

Figures 5 and 6 show streamlines and isotherms for different amplitude ratios of the sinusoidal temperature $(\varepsilon=0.5$ on the left column and $\varepsilon=1.0$ on the right

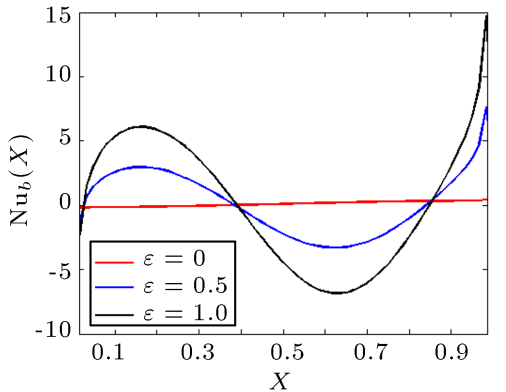

(a)

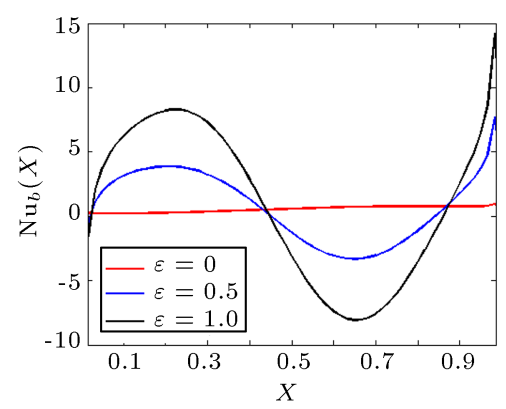

(b)

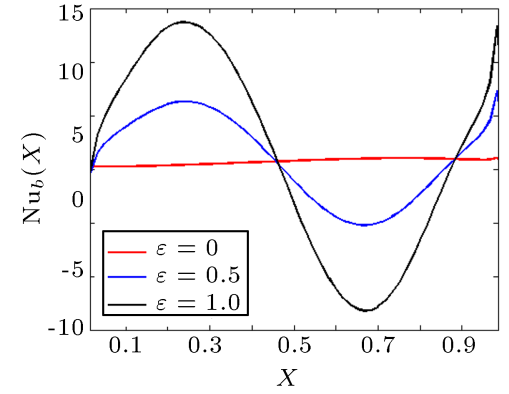

(c)

Figure 4. Local Nusselt numbers along the bottom wall for different amplitude ratios at (a) $\mathrm{Ra}=10^{3}$, (b) $\mathrm{Ra}=10^{4}$, and (c) $\mathrm{Ra}=10^{5}$ for the $\mathrm{Al}_{2} \mathrm{O}_{3}$-water nanofluid.
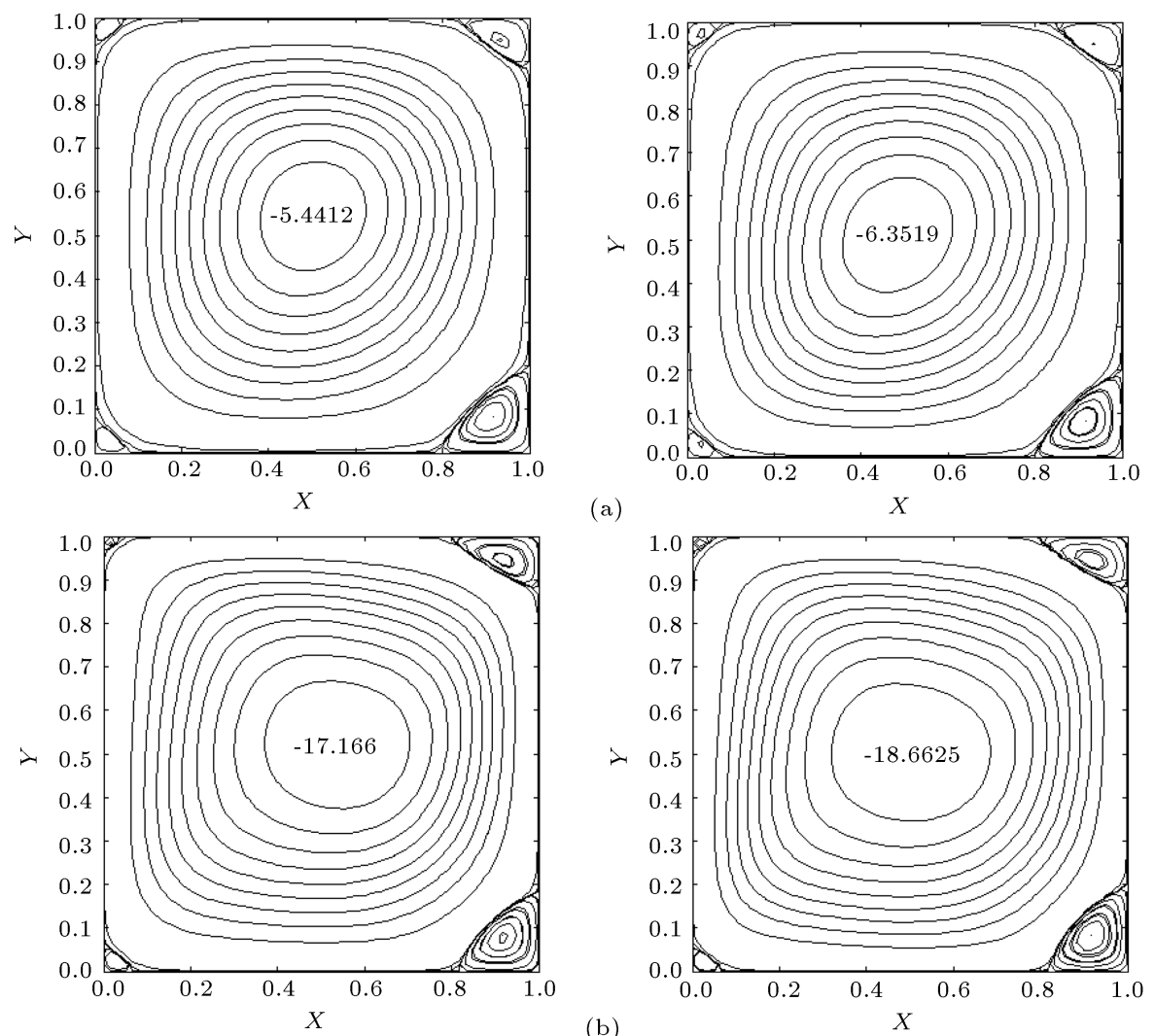

Figure 5. Streamlines for different amplitude ratios $(\varepsilon=0.5$ on the left column and $\varepsilon=1.0$ on the right column) at (a) $\mathrm{Ra}=10^{4}$ and (b) $\mathrm{Ra}=10^{5}$ for the $\mathrm{Al}_{2} \mathrm{O}_{3}$-water nanofluid. 

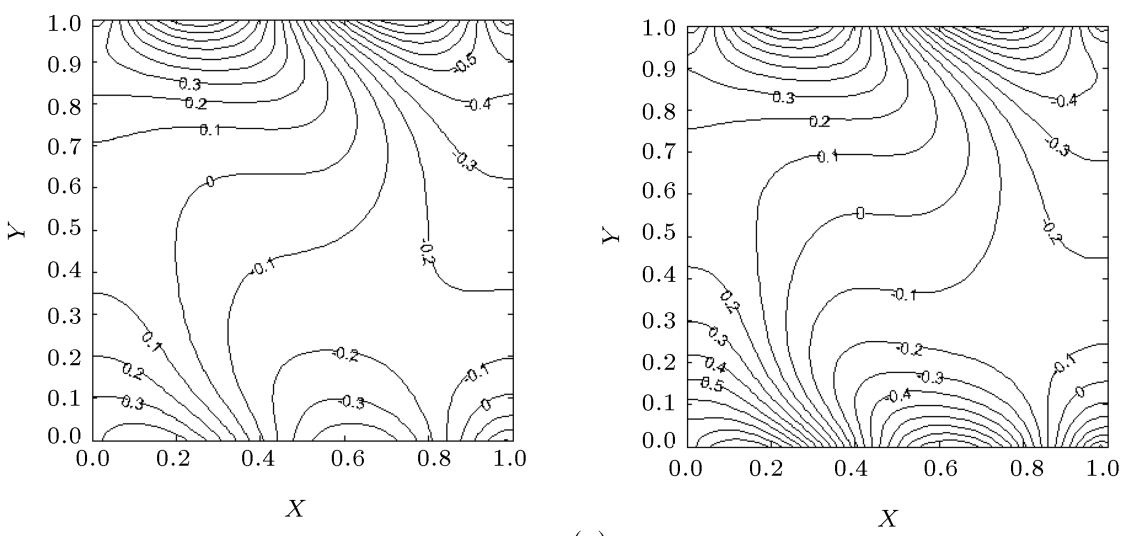

(a)
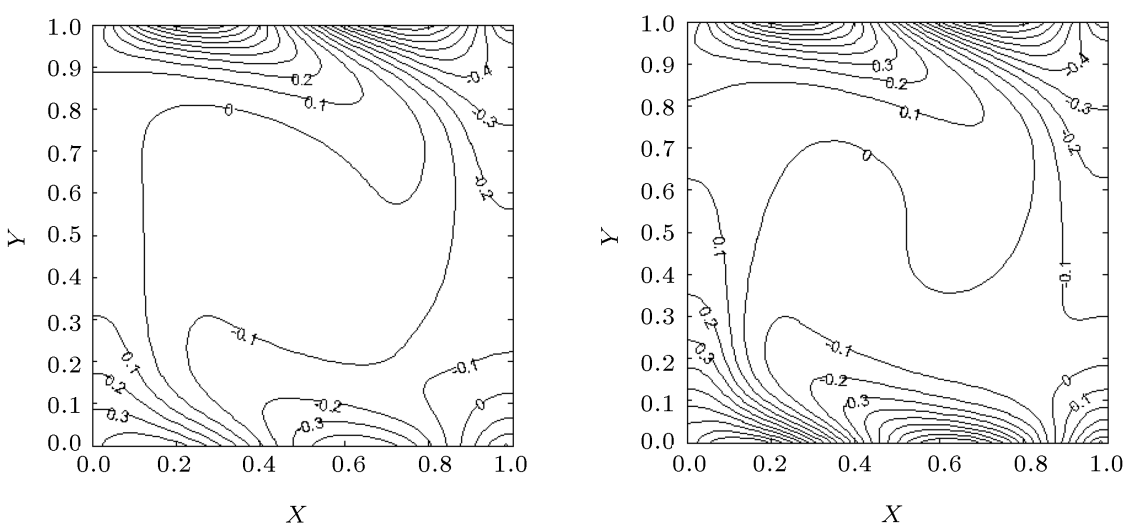

(b)

Figure 6. Isotherms for different amplitude ratios $(\varepsilon=0.5$ on the left column and $\varepsilon=1.0$ on the right column) at (a) Ra $=10^{4}$ and (b) $\mathrm{Ra}=10^{5}$ for the $\mathrm{Al}_{2} \mathrm{O}_{3}$-water nanofluid.

column) at different Rayleigh numbers for the $\mathrm{Al}_{2} \mathrm{O}_{3}$ water nanofluid with $\phi=5 \%, r=30^{\circ}$, and $\iota=\pi / 4$. Results show that with increase in $\varepsilon$ from 0.5 to 1.0 , the convective flow is distributed over the enclosure and the secondary eddy near the right-bottom corner becomes bigger and more powerful. For the same amplitude ratio, $\varepsilon$, an increase in Ra increases the value of $|\Phi|_{\max }$. For example, when $\varepsilon=0.5$, the value of $|\Phi|_{\text {max }}$ increases from 5.4412 at $\mathrm{Ra}=10^{4}$ to 17.1660 at $\mathrm{Ra}=10^{5}$. In addition, for the same Rayleigh number, an increase in $\varepsilon$ increases the value of $|\Phi|_{\max }$. For example, when $\mathrm{Ra}=10^{5}$, the value of $|\Phi|_{\max }$ increases from 17.1660 at $\varepsilon=0.5$ to 18.6625 at $\varepsilon=1.0$. From Figure 6, one may see that when $\varepsilon$ is increased from 0.5 to 1.0 , there is a significant difference in isotherms and, in particular, strong thermal boundary layers are developed along both horizontal walls. Accordingly, we conclude that increasing the amplitude ratio, $\varepsilon$, will lead to increase in heat transfer.

The effects of the phase deviation $\iota$ on the local Nusselt number along the bottom wall for $\mathrm{TiO}_{2}$-water nanofluids are demonstrated in Figure 7 , where $\phi=$ $5 \%, r=30^{\circ}$, and $\varepsilon=1.0$. From the figure, we can see that the local Nusselt number, $\mathrm{Nu}_{b}(X)$, along the bottom wall is significantly affected by changing $\iota$.
Furthermore, the heat transfer rate along the bottom wall decreases as the phase deviation increases on the left half of the square enclosure; on the other hand, it increases as the phase deviation increases on the right half of the square enclosure.

Figures 8 and 9 plot the streamlines and isotherms for different phase deviations $(\iota=\pi / 4$ on the left column and $\iota=\pi / 2$ on the right column) at different Rayleigh numbers for the $\mathrm{TiO}_{2}$-water nanofluid with $\phi=5 \%, r=30^{\circ}$, and $\varepsilon=1.0$. Results show that when the phase deviation, $\iota$, is increased from $\pi / 4$ to $\pi / 2$, the secondary vortex near the right-bottom corner becomes bigger and bigger until it is distributed over the right half of the square enclosure. Moreover, for the same phase deviation, $\iota$, one can see that an increase in Ra increases the value of $|\Phi|_{\text {max }}$. For example, when $\iota=\pi / 4$, the value of $|\Phi|_{\max }$ increases from 6.3723 at Ra $=10^{4}$ to 18.5263 at $\mathrm{Ra}=10^{5}$. On the other hand, for the same Rayleigh number, an increase in $\iota$ decreases the value of $|\Phi|_{\max }$. For example, when $\mathrm{Ra}=10^{5}$, the value of $|\Phi|_{\max }$ decreases from 18.5263 at $\iota=\pi / 4$ to 13.1169 at $\iota=\pi / 2$. From Figure 9 , we can see that both horizontal walls are divided into the heating zone and the cooling zone, because of the sinusoidal heating temperature, and the zones on the top wall are stagnant 


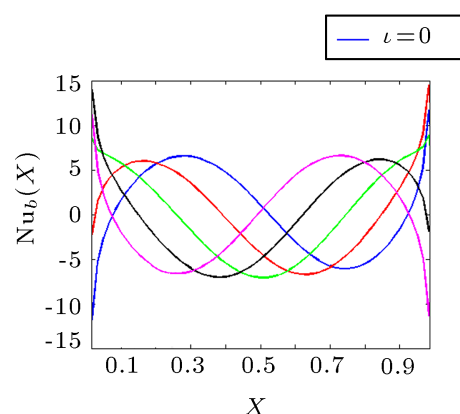

(a)

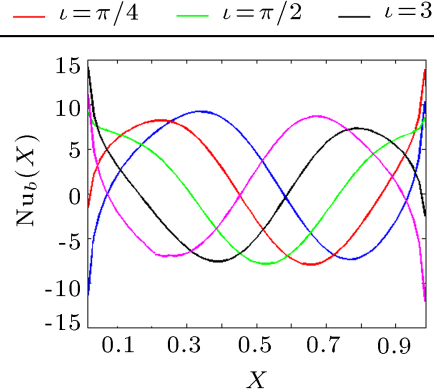

(b)

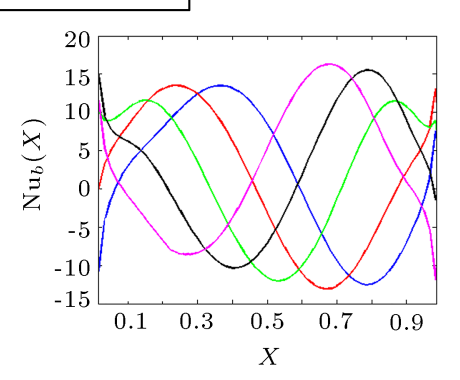

(c)

Figure 7. Effects of the phase deviation $\iota$ on the local Nusselt number along the bottom wall at $(\mathrm{a})$ Ra $=10^{3}$, $(\mathrm{b})$ Ra $=$ $10^{4}$, and (c) $\mathrm{Ra}=10^{5}$ for the $\mathrm{TiO}_{2}$-water nanofluid.
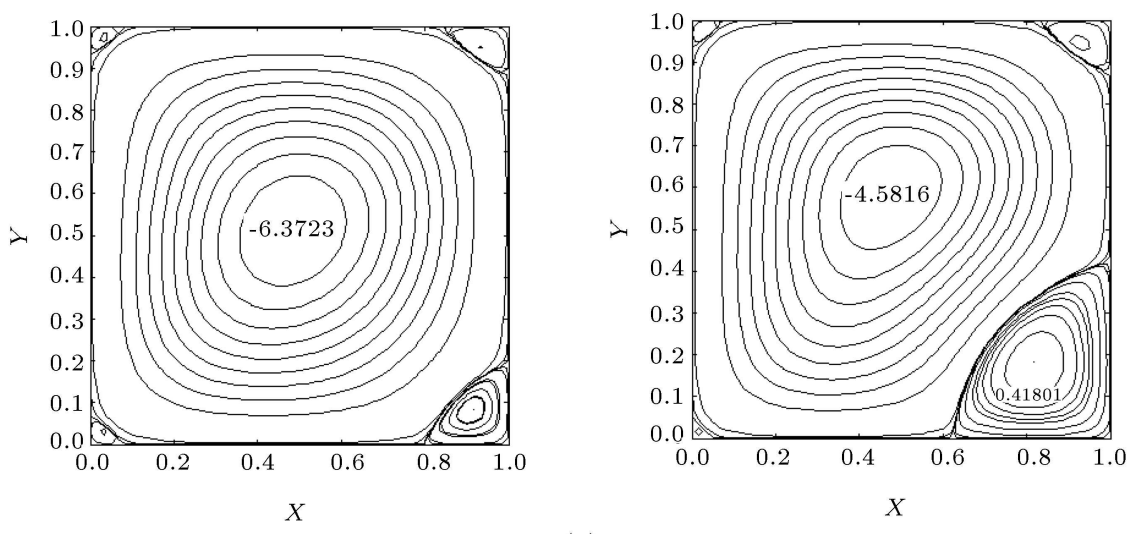

(a)
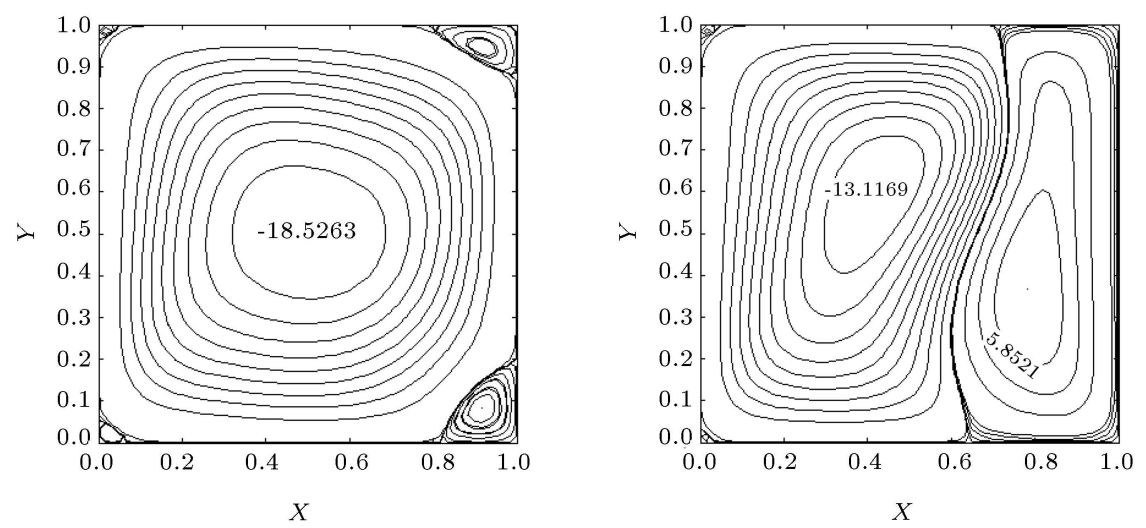

(b)

Figure 8. Streamlines for different phase deviations $(\iota=\pi / 4$ on the left column and $\iota=\pi / 2$ on the right column) at (a) $\mathrm{Ra}=10^{4}$ and (b) $\mathrm{Ra}=10^{5}$ for the $\mathrm{TiO}_{2}$-water nanofluid.

while the zones on the bottom wall shift with increase in the phase deviation.

Figures 10 and 11 demonstrate variations of the total average Nusselt number, $\mathrm{Nu}_{\mathrm{avg}}$, across the entire inclined square enclosure with different solid volume fractions for four different nanofluids $\left(\mathrm{Cu}, \mathrm{CuO}, \mathrm{Al}_{2} \mathrm{O}_{3}\right.$, $\mathrm{TiO}_{2}$ ), where $\phi=0 \%, 1 \%, 2 \%, 3 \%, 4 \%, 5 \%, r=$ $30^{\circ}, \varepsilon=0.5$, and $\iota=\pi / 4$. It is seen from these two figures that the total average Nusselt numbers are significantly affected by changing the nanoparticle volume fraction. However, the effects of the solid volume fraction parameter $\phi$ on the natural convection and heat transfer of nanofluids seem to be complicated. When the Rayleigh number $\mathrm{Ra}$ is low $\left(\mathrm{Ra}=10^{3}\right)$, the total average Nusselt number decreases as the solid volume fraction parameter, $\phi$, increases, as seen in Figure 11(a). For example, when the volume of $\mathrm{Al}_{2} \mathrm{O}_{3}$ nanoparticles increases from $0 \%$ to $5 \%$, the total average Nusselt number decreases from 0.4208 to 0.3628 at $\mathrm{Ra}=10^{3}$. On the other hand, elevating $\phi$ 

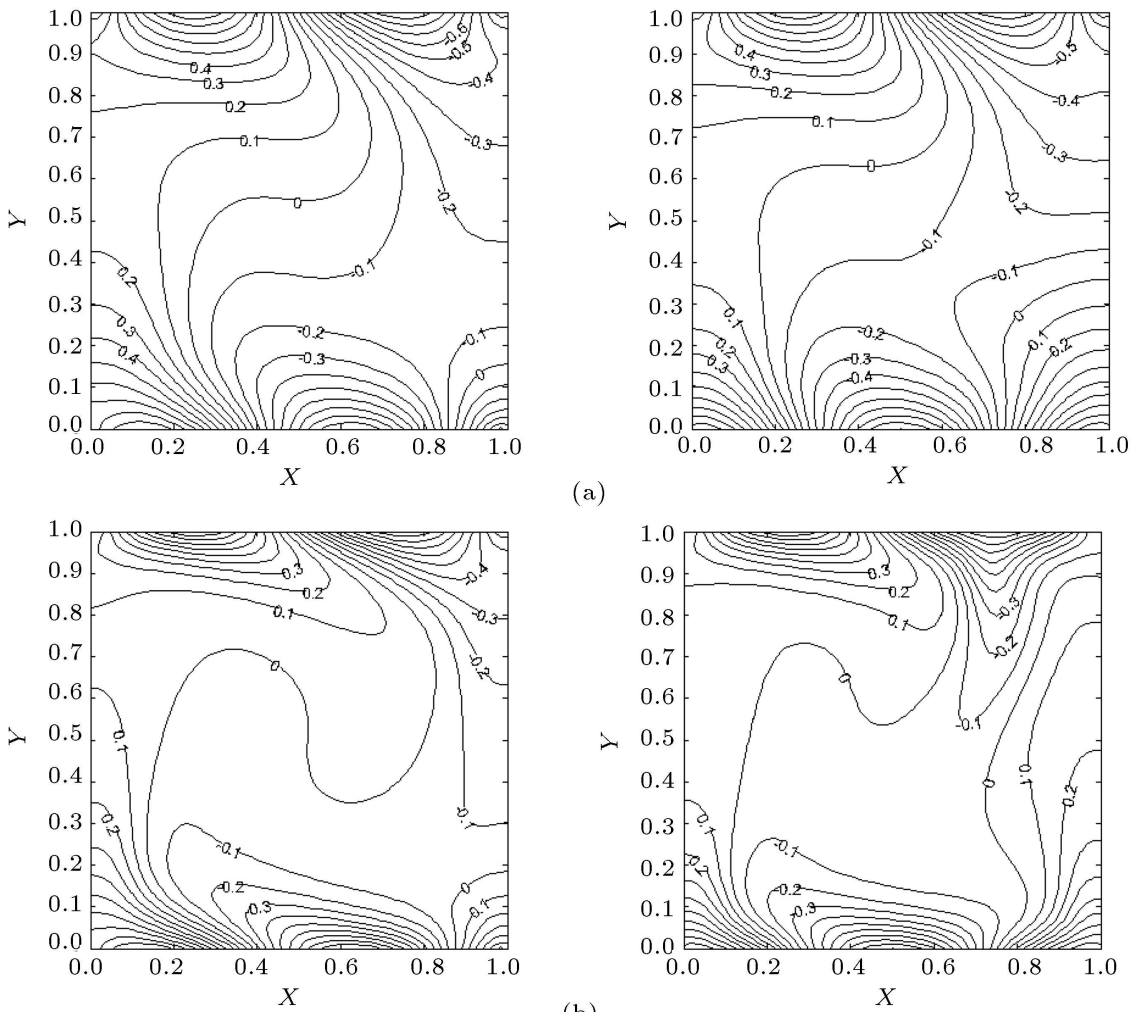

(a)

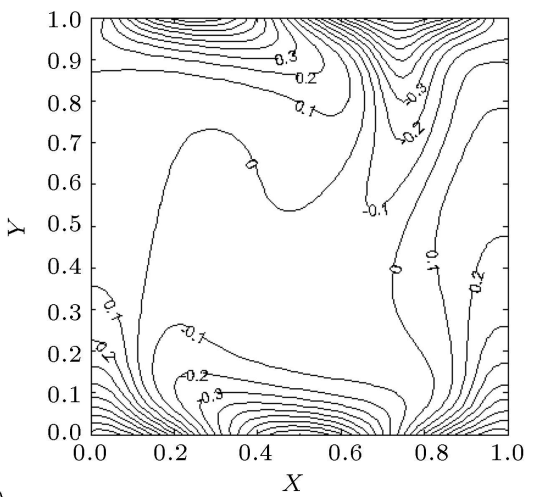

Figure 9. Isotherms for different phase deviations ( $\iota=\pi / 4$ on the left column and $\iota=\pi / 2$ on the right column) at (a) Ra $=10^{4}$ and (b) $\mathrm{Ra}=10^{5}$ for the $\mathrm{TiO}_{2}$-water nanofluid.

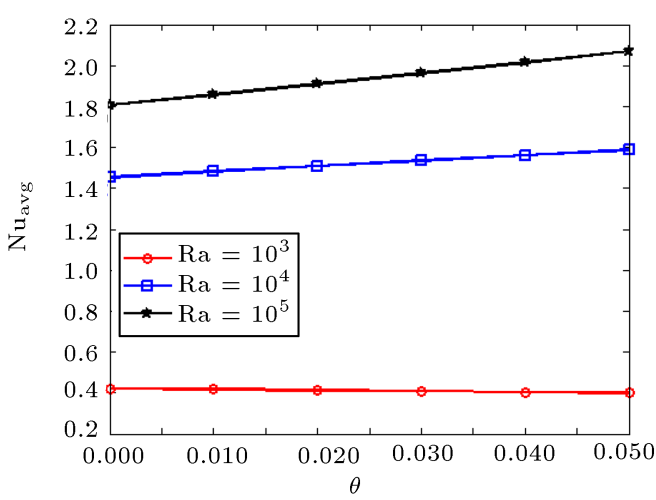

(a)

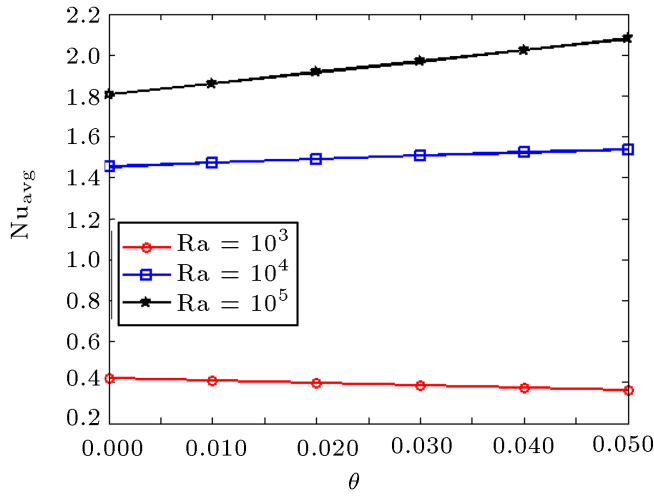

(c)

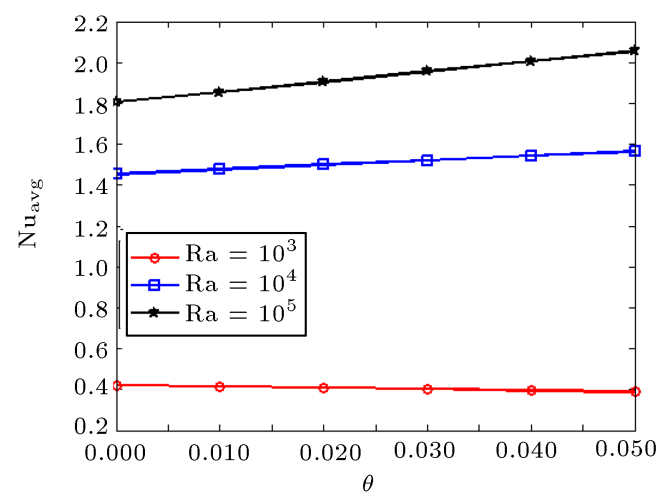

(b)

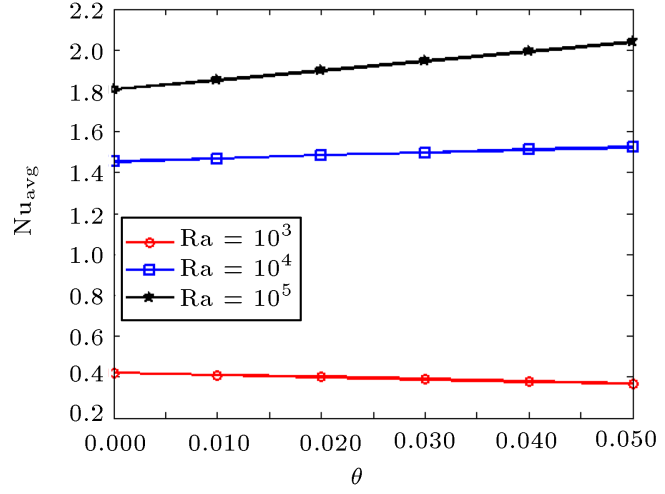

(d)

Figure 10. Variations of the total average Nusselt number across the entire inclined square enclosure with different solid volume fractions for different nanofluids of (a) $\mathrm{Cu}$, (b) $\mathrm{CuO}$, (c) $\mathrm{Al}_{2} \mathrm{O}_{3}$, and (d) $\mathrm{TiO}_{2}$. 


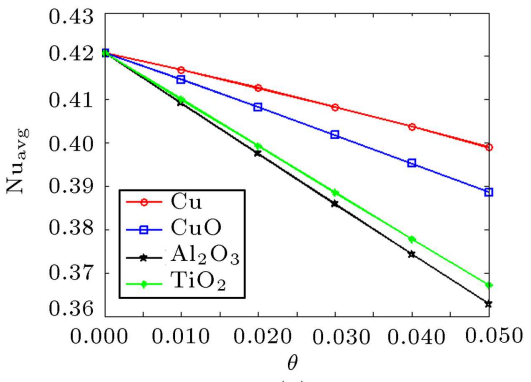

(a)

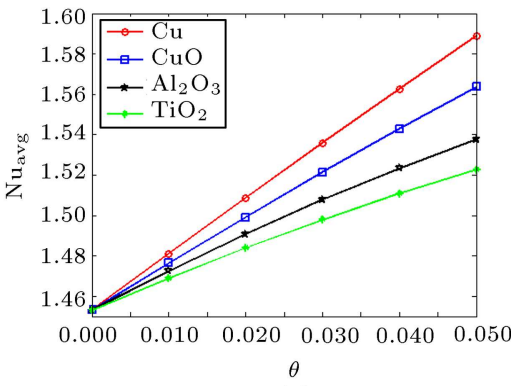

(b)

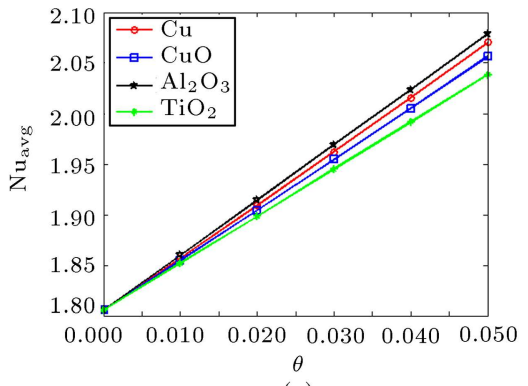

(c)

Figure 11. Variations of the total average Nusselt number with different solid volume fractions for different nanoparticles at (a) $\mathrm{Ra}=10^{3}$, (b) $\mathrm{Ra}=10^{4}$, and (c) $\mathrm{Ra}=10^{5}$.

Table 3. Nanoparticle effects on the total average Nusselt number $\mathrm{Nu}_{\mathrm{avg}}$ at various Rayleigh numbers, Ra, and the solid volume fraction, $\phi$, for four types of nanofluids.

\begin{tabular}{|c|c|c|c|c|c|c|c|c|}
\hline $\begin{array}{c}\text { Nanoparticle } \\
\text { type }\end{array}$ & $\mathbf{R a}$ & $\mathrm{Nu}_{\text {avg }}$ & $\phi=0 \%$ & $\phi=1 \%$ & $\phi=2 \%$ & $\phi=3 \%$ & $\phi=4 \%$ & $\phi=5 \%$ \\
\hline \multirow{6}{*}{$\mathrm{Cu}$} & $10^{3}$ & & 0.4208 & 0.4168 & 0.4127 & 0.4083 & 0.4037 & 0.3989 \\
\hline & & $\downarrow$ & - & $0.93 \%$ & $1.92 \%$ & $2.97 \%$ & $4.06 \%$ & $5.19 \%$ \\
\hline & $10^{4}$ & & 1.4531 & 1.4810 & 1.5086 & 1.5358 & 1.5625 & 1.5888 \\
\hline & & $\uparrow$ & - & $1.92 \%$ & $3.82 \%$ & $5.69 \%$ & $7.53 \%$ & $9.34 \%$ \\
\hline & $10^{5}$ & & 1.8056 & 1.8570 & 1.9091 & 1.9620 & 2.0158 & 2.0703 \\
\hline & & $\uparrow$ & - & $2.85 \%$ & $5.73 \%$ & $8.66 \%$ & $11.64 \%$ & $14.66 \%$ \\
\hline \multirow{6}{*}{$\mathrm{CuO}$} & $10^{3}$ & & 0.4208 & 0.4146 & 0.4083 & 0.4018 & 0.3953 & 0.3886 \\
\hline & & $\downarrow$ & - & $1.46 \%$ & $2.97 \%$ & $4.50 \%$ & $6.06 \%$ & $7.65 \%$ \\
\hline & $10^{4}$ & & 1.4531 & 1.4764 & 1.4991 & 1.5213 & 1.5429 & 1.5638 \\
\hline & & $\uparrow$ & - & $1.60 \%$ & $3.16 \%$ & $4.69 \%$ & $6.18 \%$ & $7.62 \%$ \\
\hline & $10^{5}$ & & 1.8056 & 1.8547 & 1.9043 & 1.9544 & 2.0051 & 2.0563 \\
\hline & & $\uparrow$ & - & $2.72 \%$ & $5.47 \%$ & $8.24 \%$ & $11.05 \%$ & $13.89 \%$ \\
\hline \multirow{6}{*}{$\mathrm{Al}_{2} \mathrm{O}_{3}$} & $10^{3}$ & & 0.4208 & 0.4091 & 0.3975 & 0.3858 & 0.3743 & 0.3628 \\
\hline & & $\downarrow$ & - & $2.76 \%$ & $5.53 \%$ & $8.30 \%$ & $11.05 \%$ & $13.78 \%$ \\
\hline & $10^{4}$ & & 1.4531 & 1.4725 & 1.4907 & 1.5077 & 1.5234 & 1.5376 \\
\hline & & $\uparrow$ & - & $1.33 \%$ & $2.59 \%$ & $3.76 \%$ & $4.84 \%$ & $5.82 \%$ \\
\hline & $10^{5}$ & & 1.8056 & 1.8598 & 1.9142 & 1.9688 & 2.0236 & 2.0785 \\
\hline & & $\uparrow$ & - & $3.00 \%$ & $6.02 \%$ & $9.04 \%$ & $12.08 \%$ & $15.12 \%$ \\
\hline \multirow{6}{*}{$\mathrm{TiO}_{2}$} & $10^{3}$ & & 0.4208 & 0.4100 & 0.3992 & 0.3885 & 0.3778 & 0.3672 \\
\hline & & $\downarrow$ & - & $2.56 \%$ & $5.12 \%$ & $7.67 \%$ & $10.22 \%$ & $12.74 \%$ \\
\hline & $10^{4}$ & & 1.4531 & 1.4690 & 1.4840 & 1.4980 & 1.5110 & 1.5228 \\
\hline & & $\uparrow$ & - & $1.09 \%$ & $2.12 \%$ & $3.09 \%$ & $3.98 \%$ & $4.79 \%$ \\
\hline & $10^{5}$ & & 1.8056 & 1.8519 & 1.8984 & 1.9449 & 1.9916 & 2.0383 \\
\hline & & $\uparrow$ & - & $2.56 \%$ & $5.14 \%$ & $7.72 \%$ & $10.30 \%$ & $12.89 \%$ \\
\hline
\end{tabular}

has a favorable effect on the heat transfer of nanofluids when $\mathrm{Ra}$ is high $\left(\mathrm{Ra}=10^{4}, 10^{5}\right)$, as seen in Figure 11(b) and (c). For example, when the volume of $\mathrm{TiO}_{2}$ nanoparticles increases from $0 \%$ to $5 \%$, the total average Nusselt number increases from 1.4531 to 1.5228 at $\mathrm{Ra}=10^{4}$ and increases from 1.8056 to 2.0383 at $\mathrm{Ra}$ $=10^{5}$. Based on the definitions of effective density and viscosity of nanofluids, we see that the inertial and viscous resistances of nanofluids are greater than those of water, since the density and viscosity of nanofluids are higher than those of water as $\phi$ increases. When Ra is high, the convection dominates the fluid movement. Under such circumstance, the heat transfer increases with increase in the value of the solid volume fraction parameter. Since larger volumes of nanoparticles give relatively higher thermal conductivity, it is noteworthy that high values of Ra lead to higher Nusselt numbers for each solid volume fraction. Here, we give a better comparison by examining the values presented in Table 3: 


$$
\begin{aligned}
& \uparrow=\frac{\mathrm{Nu}_{m}-\mathrm{Nu}_{f}}{\mathrm{Nu}_{f}} \times 100 \%, \\
& \downarrow=\frac{\mathrm{Nu}_{f}-\mathrm{Nu}_{m}}{\mathrm{Nu}_{f}} \times 100 \%,
\end{aligned}
$$

where $\uparrow$ and $\downarrow$ denote the increase and decrease in the total average Nusselt number of nanofluids compared to those of water, respectively [17]. It can be seen from this table that for the high values of $\mathrm{Ra}$ ( $\mathrm{Ra}$ $=10^{4}, 10^{5}$ ), the rate of change in the heat transfer rate increases by adding the volume of nanoparticles into the pure water. Furthermore, the high values of Ra are associated with the high rate of increase in the total average Nusselt number. For example, when the volume of $\mathrm{CuO}$ nanoparticles increases from $0 \%$ to $5 \%$, the rate of the total average Nusselt number increases by $7.62 \%$ at $\mathrm{Ra}=10^{4}$ and by $13.89 \%$ at $\mathrm{Ra}=10^{5}$. In addition, when $\mathrm{Ra}=10^{4}$, the value of the total average Nusselt number decreases according

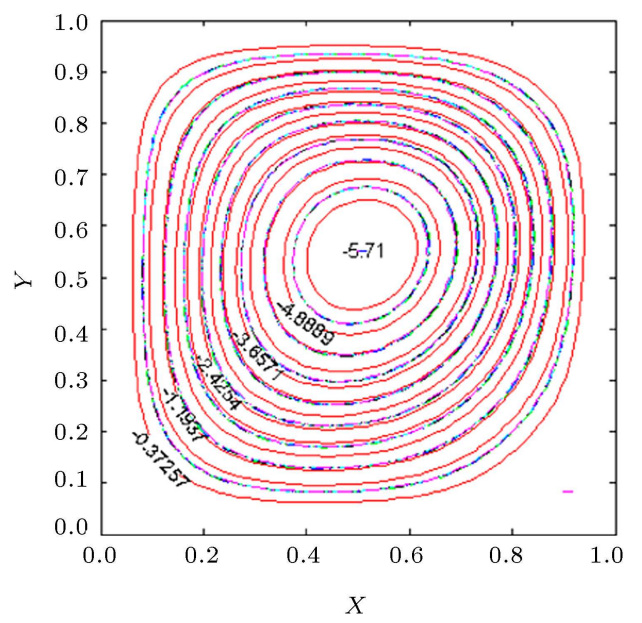

(a)

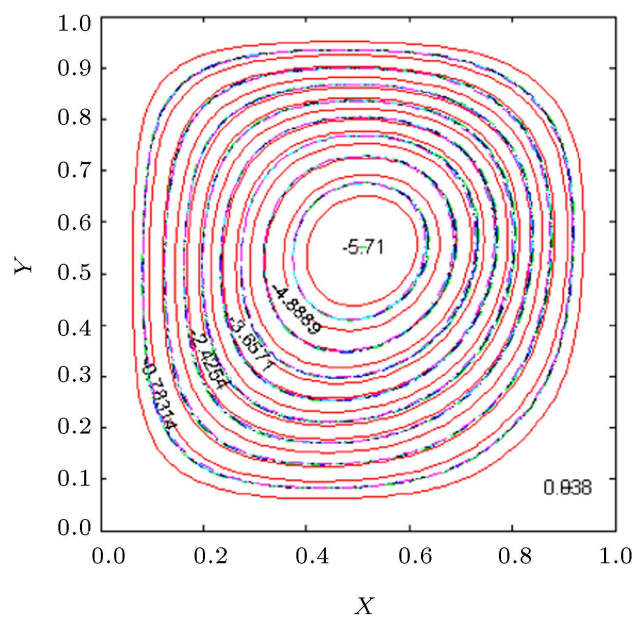

(c) to the following order of $\mathrm{Cu}, \mathrm{CuO}, \mathrm{Al}_{2} \mathrm{O}_{3}$, and $\mathrm{TiO}_{2}$. These observations are similar to those of Elif [31]. The highest heat transfer is obtained when using the $\mathrm{Al}_{2} \mathrm{O}_{3^{-}}$ water nanofluid for $\phi=5 \%$ and $\mathrm{Ra}=10^{5}$.

Figure 12 shows contours of streamlines for pure fluid $(\phi=0 \%)$ and four different nanofluids $(\phi=$ $1 \%-5 \%$ ), respectively, where $r=30^{\circ}, \varepsilon=0.5, \iota=\pi / 4$, and $\mathrm{Ra}=10^{4}$. Streamlines for nanofluids are plotted in dotted lines and those for pure fluid are plotted in red solid lines. From these streamlines, we can see that a primary recirculating cell is formed in the enclosure due to the fact that the fluid flows up near the heat source and flows down along the right and left walls with the sinusoidal temperature distribution. Furthermore, one can see that increasing $\phi$ from $1 \%$ to $5 \%$ decreases the value of $|\Phi|_{\max }$, which causes the fluid with nanoparticles to circulate slowly in the enclosure. However, the difference between pure fluid and nanofluids with four different nano-particles is negligible.

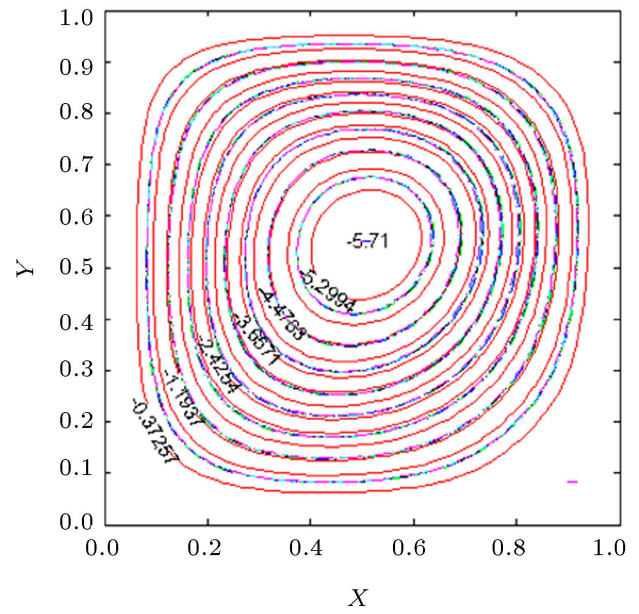

(b)

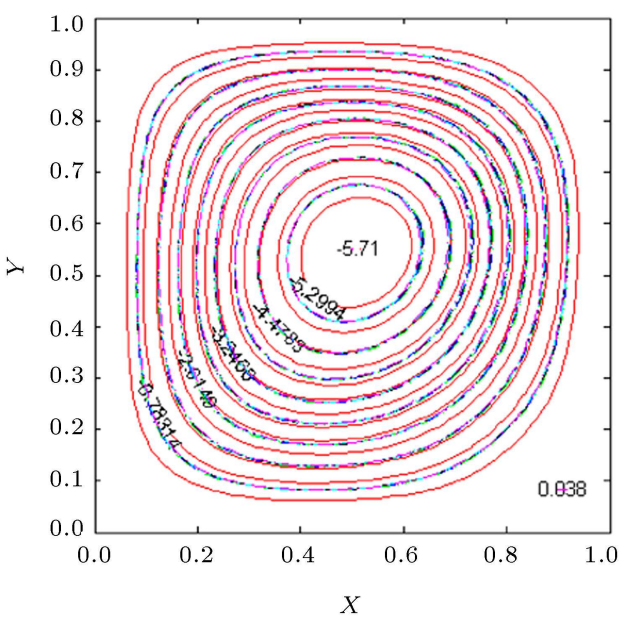

(d)

Figure 12. Streamlines for pure fluid $\phi=0 \%$ (red solid lines) and different nanofluids of (a) $\mathrm{Cu},(\mathrm{b}) \mathrm{CuO},(\mathrm{c}) \mathrm{Al}_{2} \mathrm{O}_{3}$, and (d) $\mathrm{TiO}_{2}$ with $\phi=1 \%-5 \%$ (dotted lines) at $\mathrm{Ra}=10^{4}$. 


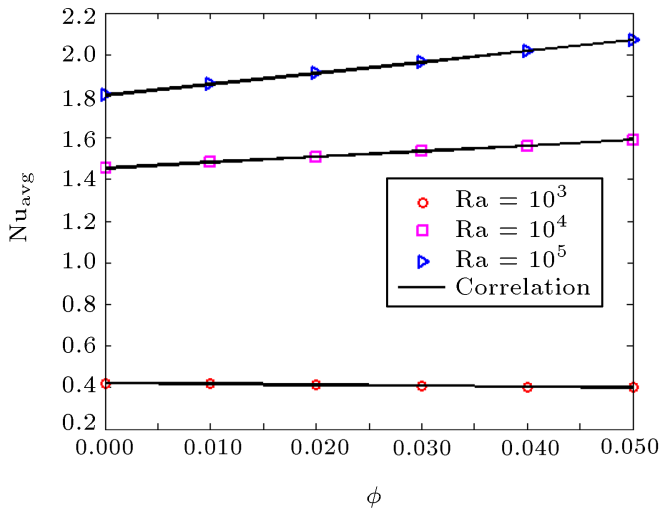

(a)

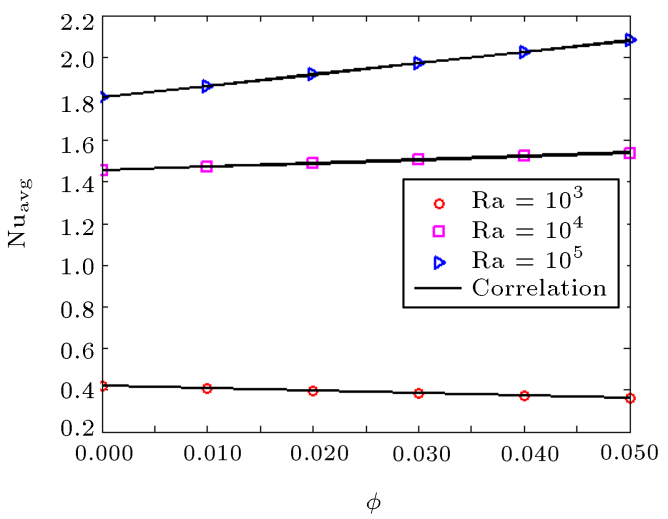

(c)

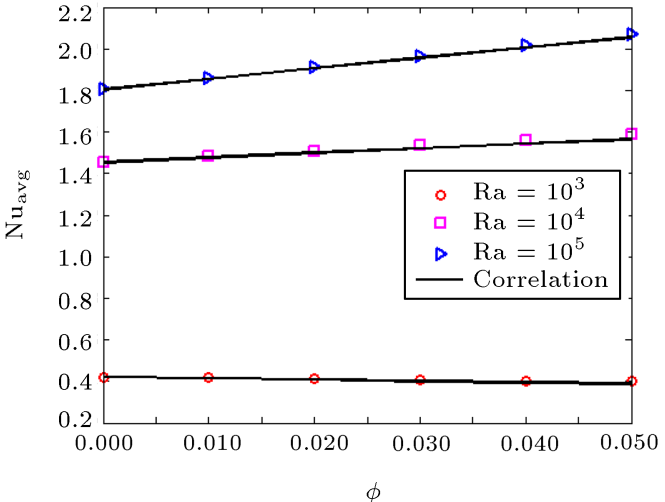

(b)

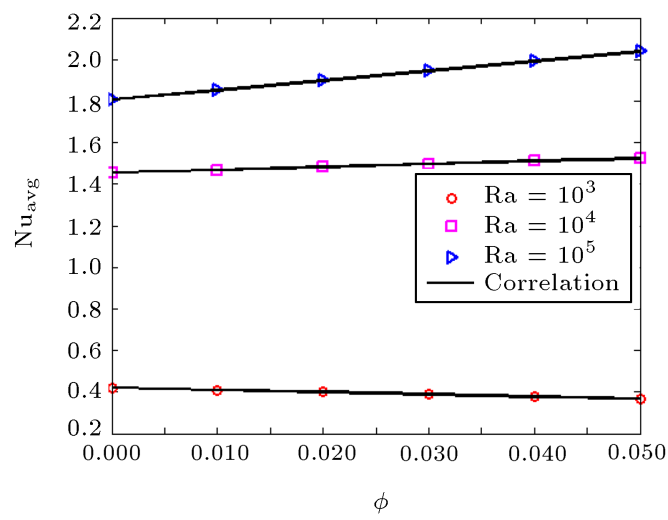

(d)

Figure 13. Comparison between the numerical results of the average Nusselt number and those obtained by the correlation in Eq. (40) for different nanofluids of (a) $\mathrm{Cu}$, (b) $\mathrm{CuO}$, (c) $\mathrm{Al}_{2} \mathrm{O}_{3}$, and (d) $\mathrm{TiO}_{2}$.

Finally, using the numerical results in Table 3 , the correlation of the total average Nusselt number along the isothermal walls can be empirically expressed as:

$$
\mathrm{Nu}_{\text {avg }}=(A+B \phi) \mathrm{Ra}^{C} \text {, }
$$

where $\phi=0 \%, 1 \%, 2 \%, 3 \%, 4 \%$, and $5 \% ; r=30^{\circ}$; $\iota=\pi / 4 ; \operatorname{Pr}=6.8$; and the corresponding coefficients and values of the squared 2-norm for the residuals are listed in Table 4. Figure 13 shows the comparison between the total average Nusselt numbers in the numerical results and those obtained based on the above correlation, which indicates that they agree well with each other.

\section{Conclusion}

We developed a numerical method for solving the natural convection and heat transfer of nanofluids in an inclined square enclosure with sinusoidal temperature distributions. The numerical scheme was derived from the FHOC finite difference method. From the obtained numerical results, we reached the following conclusions:

1. When the Rayleigh number $\mathrm{Ra}$ is low $\left(\mathrm{Ra}=10^{3}\right)$, the average Nusselt number decreases as the solid volume fraction parameter, $\phi$, increases. On the other hand, elevating $\phi$ has favorable effects on the natural convection and heat transfer of nanofluids when $\mathrm{Ra}$ is high $\left(\mathrm{Ra}=10^{4}, 10^{5}\right)$;

2. When the Rayleigh number is low $\left(\mathrm{Ra}=10^{3}\right)$, the heat transfer rates are not affected by changing the inclination angle $r$. On the other hand, when the Rayleigh number $\mathrm{Ra}$ is high $\left(\mathrm{Ra}=10^{4}, 10^{5}\right)$, the heat transfer rate decreases as the inclination angle increases;

3. For the same amplitude ratio, $\varepsilon$, an increase in $\mathrm{Ra}$ increases the value of $|\Phi|_{\max }$. In addition, for the same Rayleigh number, an increase in $\varepsilon$ also increases the value of $|\Phi|_{\max }$;

4. The phase deviation parameter has a significant impact on the local Nusselt number along the bottom wall. For the same phase deviation, an increase in Ra increases the value of $|\Phi|_{\max }$. However, for the same Rayleigh number, an increase in $\iota$ decreases the value of $|\Phi|_{\max }$;

5. When $\mathrm{Ra}=10^{4}$, the total average Nusselt number $\mathrm{Nu}_{\text {avg }}$ for the four nanofluids decreases with the 
Table 4. Corresponding coefficients and values of the squared 2-norm of the residuals for Eq. (40).

\begin{tabular}{cccccc}
\hline Nanoparticle type & $\mathbf{R a}$ & $\mathbf{A}$ & $\mathbf{B}$ & $\mathbf{C}$ & Res-norm \\
\hline $\mathrm{Cu}$ & $10^{3}$ & 10.3395 & -10.7424 & -0.4634 & $2.4586 \mathrm{E}-006$ \\
& $10^{4}$ & 9.6350 & 18.0032 & -0.2053 & $6.7317 \mathrm{E}-007$ \\
& $10^{5}$ & 9.4883 & 27.8111 & -0.1442 & $1.5336 \mathrm{E}-006$ \\
& & & & & \\
$\mathrm{CuO}$ & $10^{3}$ & 10.7493 & -16.4527 & -0.4690 & $9.1729 \mathrm{E}-007$ \\
& $10^{4}$ & 9.8626 & 15.0124 & -0.2079 & $1.3756 \mathrm{E}-006$ \\
& $10^{5}$ & 9.5192 & 26.4324 & -0.1444 & $7.0900 \mathrm{E}-007$ \\
& & & & & \\
& $10^{3}$ & 10.6119 & -29.2677 & -0.4673 & $1.1043 \mathrm{E}-007$ \\
& $10^{4}$ & 9.9221 & 11.5605 & -0.2084 & $6.8536 \mathrm{E}-006$ \\
& $10^{5}$ & 9.4655 & 28.6197 & -0.1439 & $7.6889 \mathrm{E}-008$ \\
& & & & & \\
& $10^{3}$ & 10.6115 & -27.0591 & -0.4673 & $7.8265 \mathrm{E}-008$ \\
& $10^{4}$ & 9.9675 & 9.5696 & -0.2090 & $4.3343 \mathrm{E}-006$ \\
& $10^{5}$ & 9.5585 & 24.6427 & -0.1448 & $2.71931 \mathrm{E}-008$ \\
\hline
\end{tabular}

nanoparticles in the order of $\mathrm{Cu}, \mathrm{CuO}, \mathrm{Al}_{2} \mathrm{O}_{3}$, and $\mathrm{TiO}_{2}$.

In the future work, an optimization may be needed to obtain the best value of the solid volume fraction of nanofluids in order to improve heat transfer within a porous medium in the most effective way.

\section{Acknowledgments}

This work was supported in part by the National Natural Science Foundation of China (No. U1304106).

\section{Nomenclature}

Ra Rayleigh number

$t \quad$ Phase deviation

$\mathrm{Nu} \quad$ Nusselt number

$L \quad$ Length (width) of the enclosure, $\mathrm{m}$

$A \quad$ Amplitude of sinusoidal temperature

$u, v \quad$ Velocity components, $\mathrm{m} / \mathrm{s}$

$U, V \quad$ Dimensionless velocity components

$x, y \quad$ Cartesian coordinates, $\mathrm{m}$

$X, Y \quad$ Dimensionless Cartesian coordinates

$P \quad$ Dimensionless pressure

$g \quad$ Gravitational acceleration, $\mathrm{m} / \mathrm{s}^{2}$

$r \quad$ Inclination angles

$K \quad$ Permeability of porous medium, $\mathrm{m}^{2}$

$T \quad$ Temperature, $\mathrm{K}$ $\theta \quad$ Dimensionless temperature

$\Phi \quad$ Dimensionless stream function

$\Omega \quad$ Dimensionless vorticity

Pr Prandtl number

h Mesh size

$c_{p} \quad$ Specific heat, $\mathrm{J} \mathrm{kg}^{-1} \mathrm{~K}^{-1}$

$p \quad$ Pressure, $\mathrm{N} / \mathrm{m}^{2}$

$\Psi \quad$ A transport variable

\section{Greek symbols}

$\mu \quad$ Dynamic viscosity, $\mathrm{kg} / \mathrm{ms}$

$\rho \quad$ Density, $\mathrm{kg} / \mathrm{m}^{3}$

$\phi \quad$ Volume fraction of nanoparticles

$\alpha \quad$ Thermal diffusivity, $\mathrm{m}^{2} / \mathrm{s}$

$\beta \quad$ Thermal expansion coefficient, $\mathrm{K}^{-1}$

$\gamma \quad$ Kinematic viscosity, $\mu / \rho$

$\kappa \quad$ Thermal conductivity, $\mathrm{Wm}^{-1} \mathrm{~K}^{-1}$

$\varepsilon \quad$ Amplitude ratio

$\nu \quad$ Kinematic viscosity, $\mathrm{m}^{2} / \mathrm{s}$

\section{Subscripts}

c Cold (lower value)

$f \quad$ The base fluid

avg Average

$m \quad$ Nanofluid

$s \quad$ Nanoparticle

$t \quad$ Top

$b \quad$ Bottom 


\section{References}

1. Choi, S.U.S. "Enhancing thermal conductivity of fluids with nanoparticle", Asme. Fed., 231(1), pp. 99-105 (1995).

2. Nemati, H., Farhadi, M., Sedighi, K., Ashorynejad, H.R., and Fattahi, E. "Magnetic field effects on natural convection flow of nanofluid in a rectangular cavity using the lattice Boltzmann model", Scientia Iranica, 19(2), pp. 303-310 (2012).

3. Khorasanizadeh, H., Amani, J., and Nikfar, M. "Numerical investigation of $\mathrm{Cu}$-water nanofluid natural convection and entropy generation within a cavity with an embedded conductive baffle", Scientia Iranica, 19(6), pp. 1996-2003 (2012).

4. Ho, C.J., Chen, D., Yan, W., and Wahian, O. "Buoyancy-driven flow of nanofluids in a cavity considering the Ludwig-Soret effect and sedimentation: Numerical study and experimental validation", Int. J. Heat Mass Tran., 77(4), pp. 684-694 (2014).

5. Öztop, H.F., Estellé, P., Yan, W., Al-Salem, K., Orfi, J., and Mahian, O. "A brief review of natural convection in enclosures under localized heating with and without nanofluids", Int. Commun. Heat Mass Tran., 60, pp. 37-44 (2015).

6. Esfe, M.H., Akbari, M., Karimipour, A., Afrand, M., Mahian, O., and Wongwises, S. "Mixedconvection flow and heat transfer in an inclined cavity equipped to a hot obstacle using nanofluids considering temperature-dependent properties", Int. J. Heat Mass Tran., 85, pp. 656-666 (2015).

7. Alsabery, A.I., Chamkha, A.J., Saleh, H., and Hashim, I. "Transient natural convective heat transfer in a trapezoidal cavity filled with non-Newtonian nanofluid with sinusoidal boundary conditions on both sidewalls", Powder Technol., 308, pp. 214-234 (2017).

8. Heris, S.Z., Pour, M.B., Mahian, O., and Wongwises, S. "A comparative experimental study on the natural convection heat transfer of different metal oxide nanopowders suspended in turbine oil inside an inclined cavity", Int. J. Heat Mass Tran., 73(9), pp. 231-238 (2014).

9. Rashidi, I., Mahian, O., Lorenzini, G., Biserni, C., and Wongwises, S. "Natural convection of $\mathrm{Al}_{2} \mathrm{O}_{3}$-water nanofluid in a square cavity: Effects of heterogeneous heating", Int. J. Heat Mass Tran., 74(7), pp. 391-402 (2014).

10. Li, D.F., Wang, X.F., and Hui, F. "Fully HOC scheme for mixed convection flow in a lid-driven cavity filled with a nanofluid", Adv. Appl. Math. Mech., 5(1), pp. 55-77 (2013).

11. Wang, X.F. and Wang, J.T. "Numerical simulation of natural convection in a triangle cavity filled with nanofluids using Tiwari and Das' model: Effects of heat flux", Heat Trans.-Asian Res., 46, pp. 761-777 (2017).
12. Mahian, O., Kianifar, A., Heris, S.Z., and Wongwises, S. "Natural convection of silica nanofluids in square and triangular enclosures: Theoretical and experimental study", Int. J. Heat Mass Tran., 99, pp. 792-804 (2016).

13. Estellé, P., Mahian, O., Maré, T., and Öztop, H.F. "Natural convection of CNT water-based nanofluids in a differentially heated square cavity", J. Therm. Anal. Calorim., 128(3), pp. 1765-1770 (2017).

14. Alizadeh, M.R. and Dehghan, A.A. "Conjugate natural convection of nanofluids in an enclosure with a volumetric heat source", Arab J. Sci. Eng., 39(2), pp. 1195-1207 (2014).

15. Cianfrini, M., Corcione, M., and Quintino, A. "Natural convection in square enclosures differentially heated at sides using alumina-water nanofluids with temperature-dependent physical properties", Therm. Sci., 19(10), pp. 591-608 (2015).

16. Sun, Q. and Pop, I. "Free convection in a triangle cavity filled with a porous medium saturated with nanofluids with flush mounted heater on the wall", Int. J. Therm. Sci., 50(11), pp. 2141-2153 (2011).

17. Ghasemi, B. and Aminossadati, S.M. "Mixed convection in a lid-driven triangular enclosure filled with nanofluids", Int. Comm. Heat Mass Tran., 37(8), pp. 1142-1148 (2010).

18. Mahmoudi, A.H., Shahi, M., Raouf, A., and Ghasemian, A. "Numerical study of natural convection cooling of horizontal heat source mounted in a square cavity filled with nanofluid", Int. Comm. Heat Mass Tran., 37, pp. 1135-1141 (2010).

19. Haddad, Z., Abu-Nada, E., Oztop, H.F., and Mataoui, A. "Natural convection in nanofluids: Are the thermophoresis and Brownian motion effects significant in nanofluid heat transfer enhancement?", Int. J. Therm. Sci., 57, pp. 152-162 (2012).

20. Haddad, Z., Oztop, H.F., Abu-Nada, E., and Mataoui, A. "A review on natural convective heat transfer of nanofluids", Renew. Sustain. Energy Rev., 16(7), pp. 5363-5378 (2012).

21. Donald, A.N. and Adrian, B., Convection in Porous Media, Springer, Cham (2017). https://doi.org/10.1007/978-3-319-49562-0.

22. Sivasankaran, S., Sivakumar, V., and Prakash, P. "Numerical study on mixed convection in a lid-driven cavity with non-uniform heating on both sidewalls", Int. J. Heat Mass Tran., 53(19), pp. 4304-4315 (2010).

23. Jmai, R., Ben-Beya, B., and Lili, T. "Heat transfer and fluid flow of nanofluid filled enclosure with two partially heated side walls and different nanoparticles", Superlattice Microst., 53(1), pp. 130-154 (2013).

24. Mahian, O., Kianifar, A., Kalogirou, S.A., Pop, I., and Wongwises, S. "A review of the applications of nanofluids in solar energy", Int. J. Heat Mass Tran., 57, pp. 582-594 (2013). 
25. Mahian, O., Kianifar, A., Heris, S.Z., Wen, D., Sahin, A.Z., and Wongwises, S. "Nanofluids effects on the evaporation rate in a solar still equipped with a heat exchanger", Nano Energy, 36, pp. 134-155 (2017).

26. Nasrin, R., Parvin, S., and Alim, M.A. "Heat transfer by nanofluids through a flat plate solar collector", Procedia Engineering, 90, pp. 364-370 (2014).

27. Arani, A.A.A., Sebdani, S.M., Mahmoodi, M., Ardeshiri, A., and Aliakbari, M. "Numerical study of mixed convection flow in a lid-driven cavity with sinusoidal heating on sidewalls using nanofluid", $S u$ perlattice Microst., 51(6), pp. 893-911 (2012).

28. Nasrin, R. and Alim, M.A. "Performance of nanofluids on heat transfer in a wavy solar collector", Int. J. Eng. Sci. Technol., 5(3), pp. 58-77 (2013).

29. Mejri, I. and Mahmoudi, A. "MHD natural convection in a nanofluid-filled open enclosure with a sinusoidal boundary condition", Chem. Eng. Res. Des., 98, pp. 1-16 (2015).

30. Rees, D.A.S. "The effect of steady streamwise surface temperature variations on vertical free convection", Int. J. Heat Mass Transfer, 31, pp. 1344-1353 (1999).

31. Elif, B.O. "Natural convection of water-based nanofluids in an inclined enclosure with a heat source", Int. J. Therm. Sci., 48(11), pp. 2063-2073

(2009).

32. Abu-Nada, E. "Mixed convection flow in a lid-driven inclined square enclosure filled with a nanofluid", Eur. J. Mech. B-Fluid, 29(6), pp. 472-482 (2010).

33. Ghasemi, B. and Aminossadati, S.M. "Mixed convection in a lid-driven triangular enclosure filled with nanofluids", Int. J. Heat Mass Tran., 37(8), pp. 11421148 (2010).

34. Lavasani, A., Farhadi, M., and Darzi, R. "Study of convection heat transfer enhancement inside lid driven cavity utilizing fins and nanofluid", Therm. Sci., 21(6), pp. 2431-2442 (2017).

35. Mansour, M.A., Ahmed, S.E., and Chamkha, A.J. "Entropy generation optimization for MHD natural convection of a nanofluid in porous media-filled enclosure with active parts and viscous dissipation", Int. J. Numer. Method Heat Fluid Flow, 27(2), pp. 379-399 (2017).

36. Shahriari, A., Javaran, E.J., and Rahnama, M. "Effect of nanoparticles Brownian motion and uniform sinusoidal roughness elements on natural convection in an enclosure", J. Therm. Anal. Calorim., 131, pp. 28652884 (2018).

37. Oztop, H.F. and Abu-Nada, E. "Numerical study of natural convection in partially heated rectangular enclosures filled with nanofluids", Int. J. Heat Fluid Flow, 29(5), pp. 1326-1336 (2008).

38. Ashraf, M.I. and Sinha, S. "Natural convection of nanofluids in a cavity with nonuniform temperature distributions on side walls", Numer. Heat Transfer-A Appl., 65(3), pp. 247-268 (2014).

39. Wang, X.F., Shi, D.Y., and Li, D.F. "Natural convective flow in an inclined lid-driven enclosure with a heated thin plate in the middle", Int. J. Heat Mass Tran., 55(25-26), pp. 8073-8087 (2012).

40. Erturk, E. "Numerical performance of compact fourthorder formulation of the Navier-Stokes equations", Commun. Numer. Meth. Eng., 24(12), pp. 2003-2019 (2008).

41. Erturk, E., Corke, T.C., and Gökcöl, C. "Numerical solutions of 2-D steady incompressible driven cavity flow at high Reynolds numbers", Int. J. Numer. Methods Fluids, 48(7), pp. 747-774 (2005).

42. Famouri, M. and Hooman, K. "Entropy generation for natural convection by heated partitions in a cavity", Int. Commun. Heat Mass Tran., 35(4), pp. 492-502 (2008).

43. Bejan, A. "On the boundary layer regime in a vertical enclosure filled with a porous medium", Lett. Heat Mass Tran., 6(2), pp. 93-102 (1979).

44. Gross, R.J., Bear, M.R., and Hickox, C.E. "The application of flux-corrected transport (FCT) to high Rayleigh number natural convection in a porous medium", Proc. 8th Int. Heat Tran. Conf., San Francisco, CA, pp. 2641-2646 (1986).

45. Manole, D.M. and Lage, J.L. "Numerical benchmark results for natural convection in a porous medium cavity", Heat Mass Tran. Porous Media, ASME Conf. 216, pp. 55-60 (1992).

46. Goyeau, B., Songbe, J.P., and Gobin, D. "Numerical study of double-diffusive natural convection in a porous cavity using the darcy-brinkman formulation", Int. J. Heat Mass Tran., 39(7), pp. 1363-1378 (1996).

47. Baytas, A.C. and Pop, I. "Free convection in a square porous cavity using a thermal nonequilibrium model", Int. J. Therm. Sci., 41(9), pp. 861-870 (2002).

48. Saeid, N.H. and Pop, I. "Natural convection from a discrete heater in a square cavity filled with a porous medium", J. Porous Media, 8(1), pp. 55-63 (2005).

49. Varol, Y., Oztop, H.F., and Varol, A. "Effects of thin fin on natural convection in porous triangular enclosures", Int. J. Therm. Sci., 46(10), pp. 1033-1045 (2007).

50. Talebi, F., Mahmoudi, A.H., and Shahi, M. "Numerical study of mixed convection flows in a square liddriven cavity utilizing nanofluid", Int. Commun. Heat Mass Tran., 37(1), pp. 79-90 (2010).

51. Ben-Cheikh, N., Chamkha, A.J., Ben-Beya, B., and Lili, T. "Natural convection of water-based nanofluids in a square enclosure with non-uniform heating of the bottom wall", J. Modern Phys., 4(2), pp. 147-159 (2013). 
52. Wang, X. and Dai, W. "Heatline analysis on heat transfer and convective flow of nanofluids in an inclined enclosure", Heat Tran. Eng., 39(10), pp. 843-860 (2018).

\section{Appendix A}

For a transport variable, $\psi$, the standard secondorder central difference operators within the nine-point stencil are given as [52]:

$$
\begin{aligned}
\delta_{X} \psi_{i, j}= & \frac{1}{2 h}\left(\psi_{i+1, j}-\psi_{i-1, j}\right) \\
\delta_{Y} \psi_{i, j}= & \frac{1}{2 h}\left(\psi_{i, j+1}-\psi_{i, j-1}\right) \\
\delta_{X}^{2} \psi_{i, j}= & \frac{1}{h^{2}}\left(\psi_{i+1, j}-2 \psi_{i, j}+\psi_{i-1, j}\right) \\
\delta_{Y}^{2} \psi_{i, j}= & \frac{1}{h^{2}}\left(\psi_{i, j+1}-2 \psi_{i, j}+\psi_{i, j-1}\right) \\
\delta_{X} \delta_{Y} \psi_{i, j}= & \frac{1}{4 h^{2}}\left(\psi_{i+1, j+1}-\psi_{i-1, j+1}\right. \\
& \left.-\psi_{i+1, j-1}+\psi_{i-1, j-1}\right) \\
\delta_{X}^{2} \delta_{Y} \psi_{i, j}= & \frac{1}{2 h^{3}}\left(\psi_{i+1, j+1}-2 \psi_{i, j+1}\right. \\
& \left.+\psi_{i-1, j+1}-\psi_{i+1, j-1}+2 \psi_{i, j-1}-\psi_{i-1, j-1}\right)
\end{aligned}
$$

$$
\begin{aligned}
\delta_{X} \delta_{Y}^{2} \psi_{i, j}= & \frac{1}{2 h^{3}}\left(\psi_{i+1, j+1}-2 \psi_{i+1, j}\right. \\
& \left.+\psi_{i+1, j-1}-\psi_{i-1, j+1}+2 \psi_{i-1, j}-\psi_{i-1, j-1}\right)
\end{aligned}
$$

$$
\begin{aligned}
\delta_{X}^{2} \delta_{Y}^{2} \psi_{i, j}= & \frac{1}{h^{4}}\left(\psi_{i+1, j+1}+\psi_{i-1, j+1}+4 \psi_{i, j}\right. \\
& \left.+\psi_{i+1, j-1}+\psi_{i-1, j-1}\right)-\frac{2}{h^{4}}\left(\psi_{i, j+1}+\psi_{i+1, j}\right. \\
& \left.+\psi_{i-1, j}+\psi_{i, j-1}\right) .
\end{aligned}
$$

\section{Biographies}

Xiaofeng Wang is a Professor of Mathematics at Minnan Normal University, P.R. China. He received his $\mathrm{PhD}$ in 2011 from Wuhan University, China. His research interests include natural or mixed convection, heat transfer, turbulent flow, and heat transfer of nanofluids.

Weizhong Dai is with McDermott International, Inc. and a Professor of mathematics in the College of Engineering \& Science at Louisiana Tech University, USA. His research interests include numerical methods for partial differential equations (higher-order compact schemes, finite difference, finite element, stability, and convergence), numerical heat transfer (micro/nano heat transfer and bio-heat transfer), and computational fluid dynamics. 